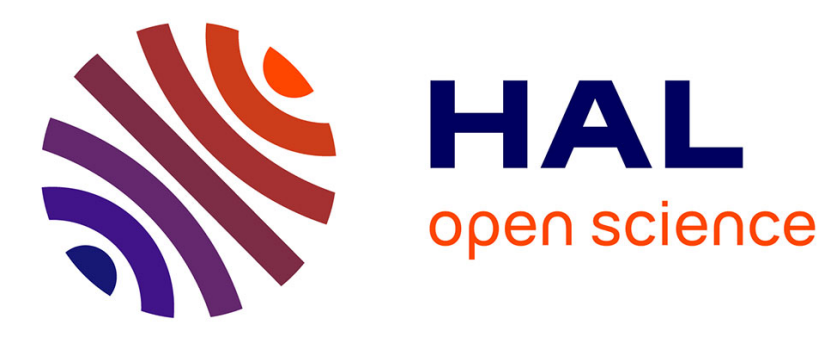

\title{
Vibration reduction of an assembly by control of the tightening load
}

\author{
Hamza Bouaziz, Nicolas Peyret, Mohamed Slim Abbes, Gael Chevallier, \\ Mohamed Haddar
}

\section{- To cite this version:}

Hamza Bouaziz, Nicolas Peyret, Mohamed Slim Abbes, Gael Chevallier, Mohamed Haddar. Vibration reduction of an assembly by control of the tightening load. International Journal of Applied Mechanics, 2016, 8 (6), 26 p. 10.1142/S1758825116500812 . hal-01480157

\section{HAL Id: hal-01480157 https://hal.science/hal-01480157}

Submitted on 1 Mar 2017

HAL is a multi-disciplinary open access archive for the deposit and dissemination of scientific research documents, whether they are published or not. The documents may come from teaching and research institutions in France or abroad, or from public or private research centers.
L'archive ouverte pluridisciplinaire HAL, est destinée au dépôt et à la diffusion de documents scientifiques de niveau recherche, publiés ou non, émanant des établissements d'enseignement et de recherche français ou étrangers, des laboratoires publics ou privés. 


\title{
Vibration reduction of an assembly by control of the tightening load
}

\author{
HAMZA BOUAZIZ ${ }^{\dagger, \ddagger, \star}$, NICOLAS PEYRET ${ }^{\ddagger, \imath}$, MOHAMED SLIM ABBES $^{\dagger, *}$, GAEL \\ $\mathrm{CHEVALLIER}^{\circledast, \boxplus}$ and MOHAMED HADDAR ${ }^{\dagger, \chi}$ \\ † Ecole Nationale d'Ingénieurs de Sfax, BP 1173-3038 Sfax - TUNISIE \\ $\ddagger$ Laboratoire QUARTZ, E7393, SUPMECA-ISMEP, 3 rue Fernand Hainaut, 93400 Saint Ouen \\ - FRANCE \\ ${ }^{*}$ Institut FEMTO-ST UMR 6174, Département Mécanique Appliquée, Université de \\ Franche-Comté, 24 Chemin de l'Epitaphe, 25000 Besançon - FRANCE \\ * hamza.bouaziz@femto-st.fr \\ 'nicolas.peyret@supmeca.fr \\ * slim.abbes@enis.rnu.tn \\ $\boxplus$ gael.chevallier@femto-st.fr \\ l Mohamed.Haddar@enis.rnu.tn \\ Received date \\ Accepted date
}

Due to their lightness, the aerospace structures are vulnerable to vibrations. Their amplitudes need to be mitigated through damping devices. The aim of this paper is to control the tightening force of a bolted joint in order to improve the vibration reduction. The work focuses on a single vibration mode modeled by two degrees of freedom coupled with a Jenkins' cell. Then two control strategies are proposed in order to reduce the harmonically forced vibrations and transient vibrations. The first control law, uses a constant optimum clamping for each excitation level. The second law consists in a sinusoidal variation of the clamping force adapted to each level and frequency of excitation. Finally, in the third section, the control law is modified in order to reduce transient vibrations. For each control law, the performances of the model are discussed according to the aimed application.

Keywords: Vibrations reduction, bolted joints, active control.

\section{Introduction}

In structures, bolted connections have been widely studied for their strength limit as well as for their interesting dynamic properties. Peyret et al. [2010] and Dion et al. [2013] compared the vibrational response of a monolithic beam with an assembled one and they showed that the interface affects the frequency response of the main structure. Indeed, partial-sliding and friction generate two effects such as a decrease of the natural frequency (variation of the stiffness) and an increase of the energy dissipation. They demonstrated the direct effect of the tightening force on the damping ratio. The field of friction control wss created thanks to both effects.

The use of friction induced damping is essential to aeronautical and aerospace structures, because they are used in environments that exceed the effectiveness temperature domain of viscoelastic materials. Nevertheless, the friction induced damping is less effective than the use of viscoelastic materials at ambient temperature 
and it behaves nonlinearly. This means that the stiffness and the damping depend on the vibration amplitude. Fig. 1 shows the influence of the excitation amplitude $F$ on the response of an assembled structure. The use of friction control is essential because of these two limitations. It aims to maximize the damping and to make it less dependent from the vibration amplitude. The first application of friction control was conducted by Lane et al. [1992].

The friction control is developed by many researchers for different applications such as the micro and nanostructures positioning, the mitigation of lightweight assembled structures vibrations or the isolation of machinery foundations vibration. Shen and Yan [2013] proposed in the first topic to overcome the uncertainties and the effect of friction of the cross roller guide in a precision positioning stage. Gaul et al. [2004] tried to suppress the vibration of large space truss structures with a semi-active approach which maximize the energy dissipation in friction joints. Stammers and Sireteanu [1998] designed a semi active device to control vibrations of machinery foundations and the ride control of a vehicle.

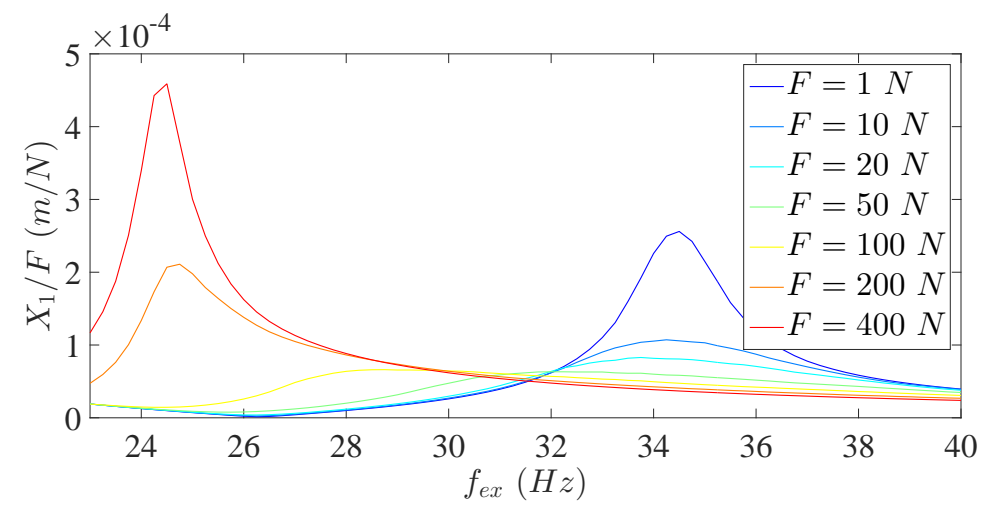

Fig. 1. View of the frequency response functions of a structure for several excitation amplitudes.

Mascarenas et al. $[2005,2006]$ emphasized on the capacity of the normal force to control and affect the behavior of the bolted joint and therefore the vibrations of the structure. In fact the normal force is the result of the tightening of the screw and the different boundary conditions of the piezoelectric patches. In addition the authors propose a health monitoring application with a low cost and a portable technology for real structures.

Dupont et al. [1997] developed the "bang-bang" control law adapted for friction, based on Lugre friction model proposed by De Wit et al. [1995]. They confirmed that the control laws can maximize the instantaneous damping induced by friction. Based on two experiments their job consists in testing a three masses system to model a turbine blade with a friction damper at the blade root. A SDOF model has also been proposed for stationary vibrations. However the proposed control laws are 
effective for only low amplitudes and low velocities in the case of impulse excitation for the first system and in the case of periodic excitation for the SDOF.

An improvement of Dupont and Stokes [1995] control law is realized by Gaul and Nitsche [2000] by increasing the instantaneous dissipated energy. They simulate and control a two-beams structure assembled with an active joint and conclude that the normal force control can substantially improve the performance of the vibration reduction compared to the constant normal force, furthermore they propose to extend the control law to an appropriate truss structure presented by Gaul et al. [1998]. The same control strategy has been adapted for a truss structure by the same authors. They tested two control laws to reduce the vibrations (see [Gaul et al., 2004] and [Gaul et al., 2005]). To obtain the friction control, L. Gaul et al. modified the original truss nodes to allow relative slip between the rods. The authors extended the concept of control previously defined for a SDOF or a 2-DOF to a multiple DOF-Systems. They used a 24 DOF reduced order model that comes form a 2523 DOF original numerical model. The 24 DOF represent 9 modes and 3 additional correction modes generated for each friction joint. In the same papers, the efficiency of the approaches for multiple MDOF-Systems modal control is demonstrated for impulse excitation.

Karim and Blanzé [2014] tested numerically two control laws for a 4-DOF system which models two bolted beams. The first control law consists in reducing the normal force of the structure in the critical frequencies vicinity. The second control law consists in varying harmonically the normal force. Furthermore, they proved that the dissipated energy maximization is an inopportune criterion and causes the highest vibration amplitudes. They confirmed also that reducing the vibration amplitude by a tightening control law could cause a global loss of the rigidity and affect the integrity of the structure. Thus they proposed an interesting dynamic absorber bolted ("add-on bolt") to the structure in order to overcome these constraints. However using the laws control cited previously for the "add-on bolt" is a difficult challenge.

In this work, we work on a 2-DOFs model able to reproduce one of the vibration modes of a real structure with an active connection. The first aim of this model is to clarify the relationship between the vibration amplitudes, the critical frequencies, the excitation levels and the tightening force. The second aim is to build a new friction control law with a variable tightening force. For the 2-DOF model, three laws are proposed in order to control forced and free vibrations. The joint between both coupled masses is modeled by a spring-dashpot linear link and a Jenkins' cell governed by Dahl's model [1968]. The control of the normal force (tightening force) may be performed by electrically commanded piezoelectric devices. We propose two new control laws for the steady state of the periodic vibration. Indeed we consider only the vibration amplitudes at resonance since the vibrations elsewhere are very low. The first control law consists in applying an optimum constant tightening force which depends on the excitation level. This control law is an improvement 
H. Bouaziz et al.

of the one proposed by Karim and Blanzé [2014] witch is switching between two levels of tightening force. A sinusoidal tightening law is introduced as the second law so called "phase control law". The sinusoidal tightening aims to eliminate the discontinuity of the square signal that leads to chattering. The limit values and the frequency of the proposed control law depend on the excitation frequency and the vibration level respectively. We propose to complete the control law designed for steady state vibrations in order to also control transient vibrations by using the study of Gaul and Nitsche [2000]. The paper starts by the formulation of a 2-DOFs system and a design of experiments on its parameters that allows us to choose the system parameters. Then, several control laws are discussed in term of efficiency for both periodic and transient excitations.

\section{Two DOF system with coupling Jenkins}

\subsection{Formulation}

The 2-DOFs coupled with a Jenkins' cell, Fig. 2, is the simplest model to study the influence of friction induced damping in a jointed structure. This simple model can be identified from the modal parameters of the Finite Element Model of a more complex structure including the bolted joints, see [Festjens et al., 2014].

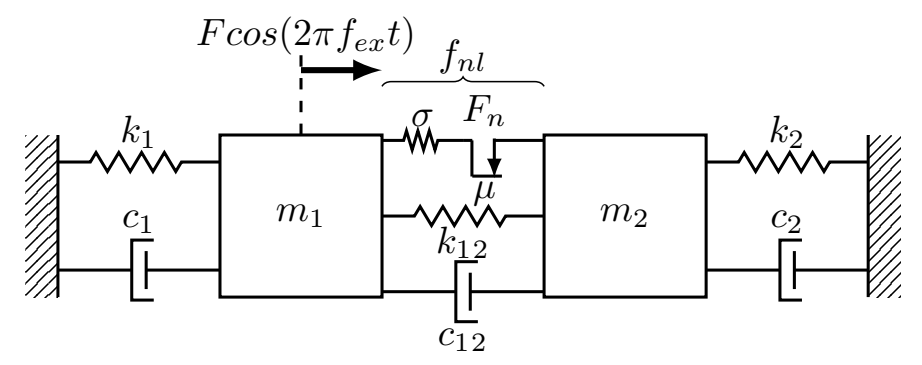

Fig. 2. Model of two degrees of freedom system

In this model, $k_{i}$ and $c_{i}(\mathrm{i}=1,2)$ are respectively the modal stiffness and the viscous damping attached to the modal mass $m_{i}$ and to the ground. $k_{12}$ and $c_{12}$ are respectively the stiffness and the viscous damping attached to both masses. $f_{n l}$ represents a modal Jenkins' cell, as described by Deaner et al. [2015], whose parameters are the coupling stiffness $\sigma$, the tightening force $F_{n}$ and the friction coefficient $\mu$.

The Jenkins' cell modelizes the partial and the total sliding phases in the interfaces of the bolted assembled structure. The stiffness $k_{12}$ is added to the coupling stiffness $\sigma$ because even in the total sliding phase, there is always a stiffness which connects both masses as demonstrated by Gaul and Lenz [1997]. Otherwise, the 
mode coupling tends to zero in full sliding. This is never observed for bending modes. According to Fig. 2, the equation of motion is given by:

$$
\begin{aligned}
{\left[\begin{array}{cc}
m_{1} & 0 \\
0 & m_{2}
\end{array}\right]\left(\begin{array}{l}
\ddot{x}_{1} \\
\ddot{x}_{2}
\end{array}\right)+\left[\begin{array}{cc}
c_{1}+c_{12} & -c_{12} \\
-c_{12} & c_{2}+c_{12}
\end{array}\right]\left(\begin{array}{l}
\dot{x}_{1} \\
\dot{x}_{2}
\end{array}\right)+} & {\left[\begin{array}{cc}
k_{1}+k_{12} & -k_{12} \\
-k_{12} & k_{2}+k_{12}
\end{array}\right]\left(\begin{array}{c}
x_{1} \\
x_{2}
\end{array}\right)=} \\
& \left(\begin{array}{c}
F \cos \left(2 \pi f_{e x} t\right) \\
0
\end{array}\right)+\left(\begin{array}{c}
-f_{n l} \\
f_{n l}
\end{array}\right) .
\end{aligned}
$$

$x_{i}, \dot{x}_{i}$ and $\ddot{x}_{i}$ are respectively the displacement, the velocity and the acceleration of the mass $m_{i}$ and $F \cos \left(2 \pi f_{e x} t\right)$ is the excitation force applied to the first mass. Dahl's friction model [1968] is used for the jenckin's cell because to our point of view it can be easily implemented in time-integration algorithms and it includes different aspects of friction such as partial and full sliding. $f_{n l}$ is thus defined by:

$$
\frac{d f_{n l}}{d \delta}=\sigma\left(1-\frac{f_{n l}}{F_{c}} \operatorname{sign}(\dot{\delta})\right)^{\alpha},
$$

where $\delta=x_{2}-x_{1}$ is the relative displacement between both masses, $F_{c}=\mu F_{n}$ represents Coulomb's sliding friction force and $\alpha$ is the constant that determines the shape of the friction law, see Fig. 3. Bliman [1992] showed that the brittle or ductile material type determines the magnitude of this parameter.

The force-displacement graph is independent from the modal parameters as mentioned by Pennestri et al. [2007]. It only depends on Dahl's parameters, Fig. 3. Moreover, the hysteresis loop area and $\delta_{\max }$ are almost independent from the parameter $\alpha$. On the other hand, the strain energy decreases following $\alpha$ increase. This proves the influence of the parameter $\alpha$, i.e. the interface topology, on the friction induced damping.

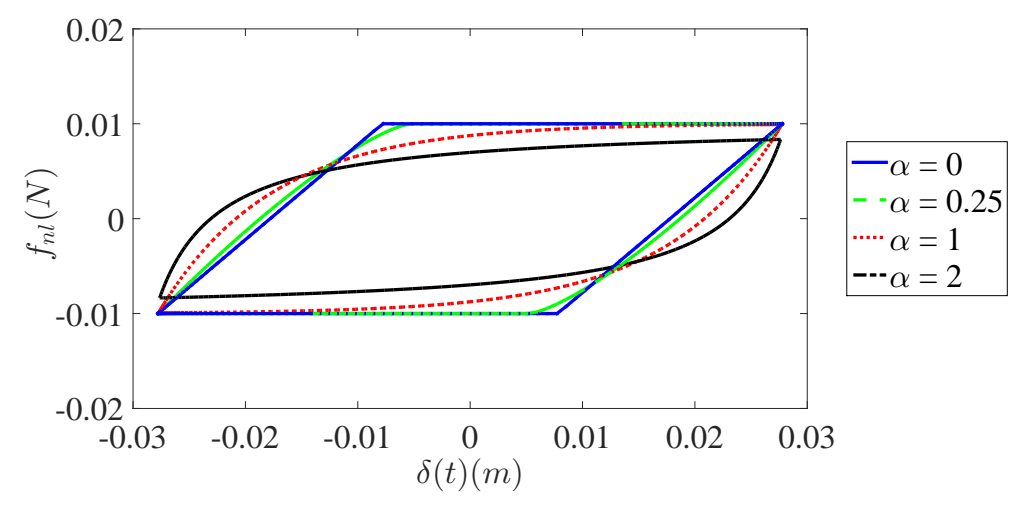

Fig. 3. $f_{n l}(\alpha)$ when $\sigma=1 N / m, F=1 N, F_{c}=0.01 N$ 


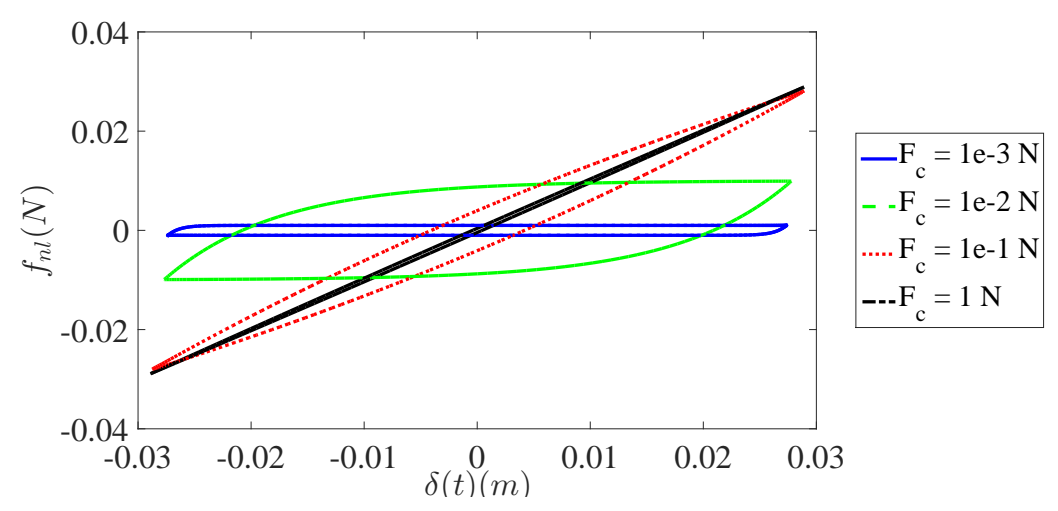

Fig. 4. $f_{n l}\left(F_{c}\right)$ when $\sigma=1 N / m, F=1 N, \alpha=1$

The energy dissipated by friction can be estimated by the hysteresis loop area, see Fig. 4. Raising the excitation frequency increases the dissipated energy until it reaches a maximum then it decreases (see Fig. 10). $f_{n l}$ tends to be a simple spring when the tightening force $F_{n}$ is important. The evolution of the energy dissipated by friction predicts that there is an optimum tightening force when the damping induced by friction occurs is maximum.

In subsequent sections we adapt a symmetric 2-DOF system $\left(k=k_{1}=k_{2}\right.$, $m=m_{1}=m_{2}$ and $\left.c=c_{1}=c_{2}\right)$ and we assume that $\sigma=1.5 k_{12}$.

\subsection{Coupling coefficient of a bolt connection}

The coupling coefficient of a bolt connection might be seen as an energetic ratio that indicates the ability of the joint to influence the vibration of the structure. It is very similar to the notion of electromechanical coupling in active systems, see [Chevallier et al., 2008] and to the notion of joint coupling level for viscoelastic materials, see [Hammami et al., 2016]. This coefficient can also be used for structures with bolted joints and can be helpful for the design of assemblies. In order to be influential, the control law of the tightening force may induce a change of the resonance frequencies. To highlight the coupling, the previous model, Eq. (1), is written assuming that the slider is sticking. This leads to a linear set of equations where the stiffness matrix is augmented by the friction linearized stiffness $\sigma$.

$$
\begin{aligned}
& {\left[\begin{array}{cc}
m & 0 \\
0 & m
\end{array}\right]\left(\begin{array}{l}
\ddot{x} \\
\ddot{y}
\end{array}\right)+\left[\begin{array}{cc}
c+c_{12} & -c_{12} \\
-c_{12} & c+c_{12}
\end{array}\right]\left(\begin{array}{l}
\dot{x} \\
\dot{y}
\end{array}\right)+} \\
& {\left[\begin{array}{cc}
k+k_{12}+\sigma & -k_{12}-\sigma \\
-k_{12}-\sigma & k+k_{12}+\sigma
\end{array}\right]\left(\begin{array}{l}
x \\
y
\end{array}\right)=\left(\begin{array}{l}
0 \\
0
\end{array}\right) \text {. }}
\end{aligned}
$$

A modal viscous damping equal to $1 \%$ for both modes is added to the model to take into account the other dissipation sources. There is no justification for 
this damping value except that it is often found experimentally fo this kind of structure. Anyway, the results below do not lose generality if this value changes moderately. Then the modal damping matrix is converted into viscous dampers using the eigenmodes:

$$
\xi=1 \% \Rightarrow C=\left(\begin{array}{cc}
c+c_{12} & -c_{12} \\
-c_{12} & c+c_{12}
\end{array}\right)=\varphi^{\prime}\left(\begin{array}{cc}
2 \xi \omega_{0,1} & 0 \\
0 & 2 \xi \omega_{0,2}
\end{array}\right) \varphi
$$

The eigenvalues, in Laplace domain, of the linearized system (3) are equal to:

$$
s_{i}=-\xi_{i} \omega_{0, i} \pm j \omega_{0, i} \sqrt{1-\xi_{i}^{2}}
$$

According to the numerical values, the first pulsation $\omega_{0,1}$ is rather independent from the coupling stiffness whereas the second pulsation $\omega_{0,2}$ strongly depends on the coupling stiffness:

$$
f_{0,2}=\frac{\omega_{0,2}}{2 \pi}=\frac{\sqrt{\frac{k+2 k_{12}}{m}}}{2 \pi} .
$$

From a physical point of view, applying a large enough tightening force reduces the system to a 2 -DOFs linear system coupled by $k_{12}+\sigma$. However canceling the tightening force reduces the system to a 2 -DOFs linear system only coupled by $k_{12}$. Therefore the coupling coefficient $K_{2}$ is defined as:

$$
K_{2}^{2}=\frac{\Delta f_{0,2}^{2}}{f_{0,2}^{2}}=\frac{2 \sigma}{k+2 k_{12}} .
$$

The sensitivity of the coupling coefficient to the stiffnesses $k$ and $k_{12}$ is plotted on Fig. 5, when the mass $m$ is set to $1 \mathrm{~kg}$ and $\sigma=1.5 k_{12}$. A large value of $k$ and a small value for $k_{12}$ and $\sigma$ leads to the best compromise.

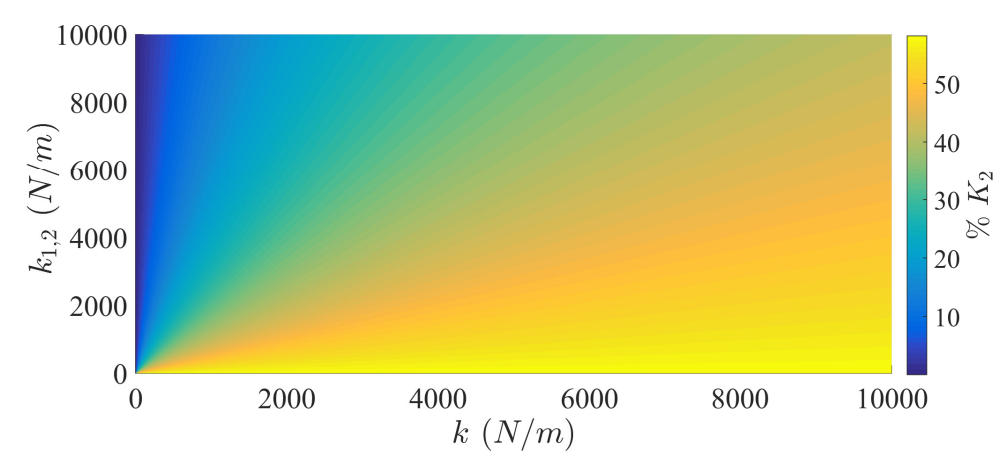

Fig. 5. Coupling coefficient of the $2^{\text {nd }}$ mode according to $k_{1}$ and $k_{12}$ stiffnesses. 
Considering the nonlinear system, Eqn. 1 , the resonance frequency $f_{r, 2}$ is very sensitive to the tightening force $F_{c}$ and to the excitation amplitude $F$, see Fig. 6 . The definition of the coupling coefficient is extended to the non-linear system to the tightening and to the excitation force, see Fig. 7. The evolution of the second resonance frequency Fig. 6, according to the excitation force is bounded by the natural frequencies of the linearized system when $\sigma=1.5 k_{12}$ and when $\sigma=0$. For each level of tightening, the resonance frequency decreases linearly until it reaches the minimum value (i.e. when $\sigma=0$ ), and then it stabilizes at this value. The smaller the tightening force, the greater the downward slope and the greater effect on the vibration amplitudes.

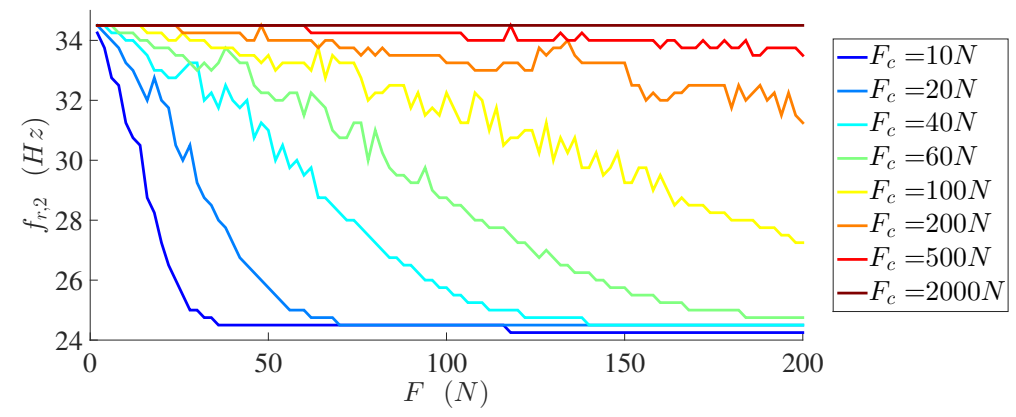

Fig. 6. Frequency of the second resonance as a function of the excitation force $F$ and the tightening force $F_{c}$.

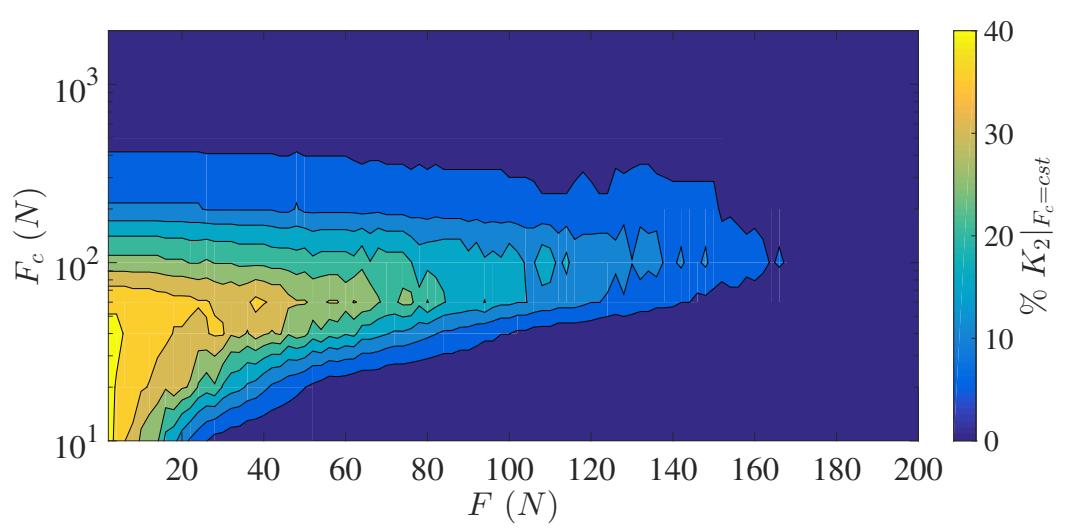

Fig. 7. Coupling coefficient of the $2^{\text {nd }}$ mode according to $F$ and $F_{c}$. 


\section{Control strategies for steady state vibrations}

Karim and Blanzé [2014] showed that the energy dissipated by friction tends to a maximum when the displacement tends to its maximum. Thus, for this reason and because it is easier to observe the vibration amplitude than the dissipated energy, the vibration amplitude is chosen as the observation state. Three ideas are evaluated in order to attenuate the vibration amplitude when the system is harmonically forced, Fig. 8:

- From Fig. 9a, one can observe that the resonance frequency $f_{r, 2}$ strongly depends on the tightening force. In this case, for a given excitation amplitude, it shifts from $24 \mathrm{~Hz}$ to $34 \mathrm{~Hz}$ when the tightening force is respectively equal to $2 N$ and $200 N$. According to these observations, the first control strategy is to change the tightening force according to the excitation frequency in order to shift the resonant frequency and to avoid the resonance phenomena. It is illustrated on Fig. 8a and b, where the first control law is a constant tightening load over the time range equal to $200 \mathrm{~N}$ for the lowest excitation frequency and to $2 N$ for the highest excitation frequency. This control strategy has been used by Karim and Blanzé [2014] in their work.

- From Fig. 9a and from Fig. 1, one can observe that the vibration amplitude depends on the excitation frequency and on the excitation amplitude. Moreover, for a given vibration amplitude, there is an optimal tightening load available over the excitation frequency range. If $F=100 N, F_{c}$ is equal to $60 N$. Thus, the second strategy consists in applying an optimum constant tightening force $F_{c_{o p t}}$ according to the vibration amplitude. This leads to the lowest amplitude vibration at the resonance and the largest damping rate at the resonance frequencies.

- The third strategy of control is very close to the switching control, see for instance [Badel et al., 2006]. As the resonance frequency depends on the tightening force, the idea is to change continuously the tightening load in order to avoid the resonance phenomena. Practically, the tightening force is harmonically changing. The parameters, frequency, phase, amplitude are chosen in order to get the lowest vibration amplitude at each frequency and amplitude of the excitation force.

\subsection{First control strategy}

The effect of the tightening force on the frequency response function is highlighted on Fig. 9. Switching between $F_{c}=2 N$ when the excitation frequency $f_{e x}$ is greater than the switching frequency, and $F_{c}=2000 N$ when $f_{e x}$ is lower than the switching frequency, ensure the lowest amplitude of each excitation frequency. Unfortunately, as presented by Karim and Blanzé [2014], the switching frequency depends on the excitation amplitudes. Therefore it is necessary to provide either a numerical or 


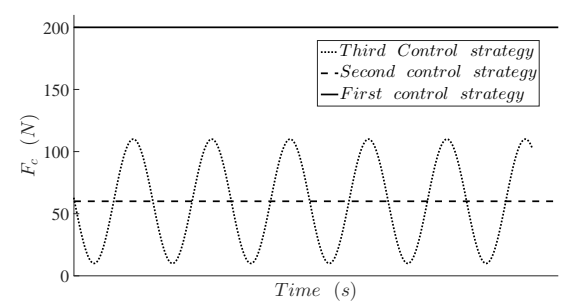

(a)

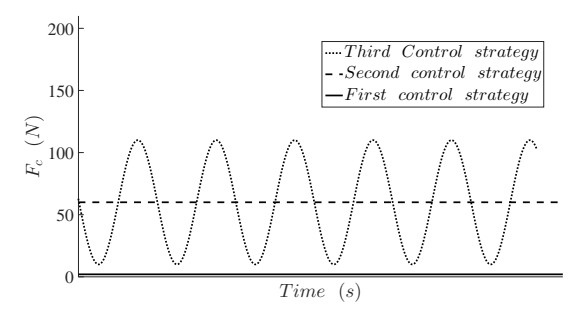

(b)

Fig. 8. Comparison of the tightening forces within the three control strategies when $F=100 \mathrm{~N}$ and (a) $f_{e x}=28 \mathrm{~Hz}$ and (b) $f_{e x}=34 \mathrm{~Hz}$.

an analytical chart in order to give the optimal tightening force at each level of excitation force.

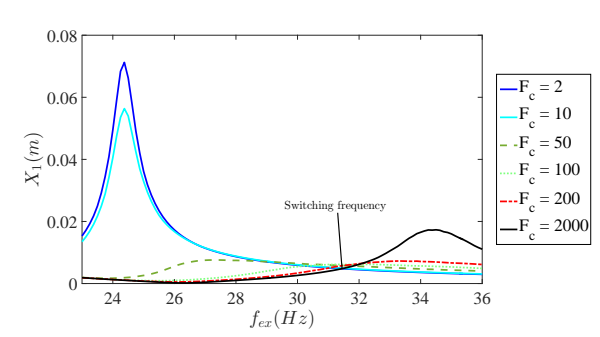

(a)

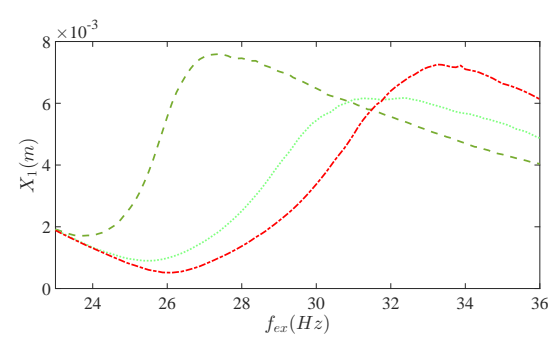

(b)

Fig. 9. (a) Vibration amplitude of the first mass around the second resonance frequency for several tightening levels when the excitation force $F$ is equal to $100 N$, (b) zoom on the lowest levels

\subsection{Second control strategy}

Knowing the first harmonic of the displacement field, the equivalent damping ratio $\xi_{e q}$ can be computed using the following definition:

$$
\xi_{e q}=\frac{W_{d i s}}{4 \pi E_{p}},
$$

where $W_{d i s}$ is the energy dissipated by friction in the joints under harmonic loadings. Graphically, $W_{d i s}$ is the area of the hysteresis loop in the force-displacement map. Whereas $E_{p}$ is the maximum strain energy over the cycle:

$$
E_{p}=\frac{1}{2} k_{1} x_{1_{\text {max }}}^{2}+\frac{1}{2} k_{2} x_{2_{\text {max }}}^{2}+\frac{1}{2}\left(k_{12}+\sigma\right)\left(x_{2}-x_{1}\right)_{\max }^{2} .
$$

Considering the numerical values mentioned above, $\xi_{e q}$ is plotted according to the frequency $f_{e x}$ and to the tightening force $F_{c}$, see Fig. 12 . In the case $F_{c}=40 \mathrm{~N}$ 


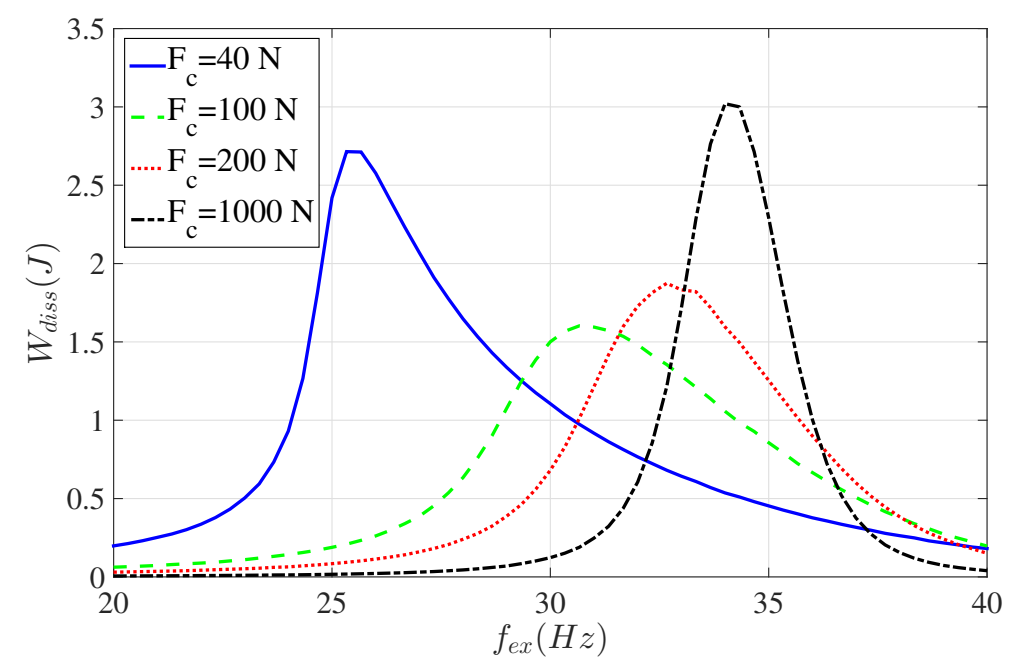

Fig. 10. Energy dissipated per cycle in steady state versus the excitation frequency for 4 tightening levels when the excitation force $F=100 \mathrm{~N}$.

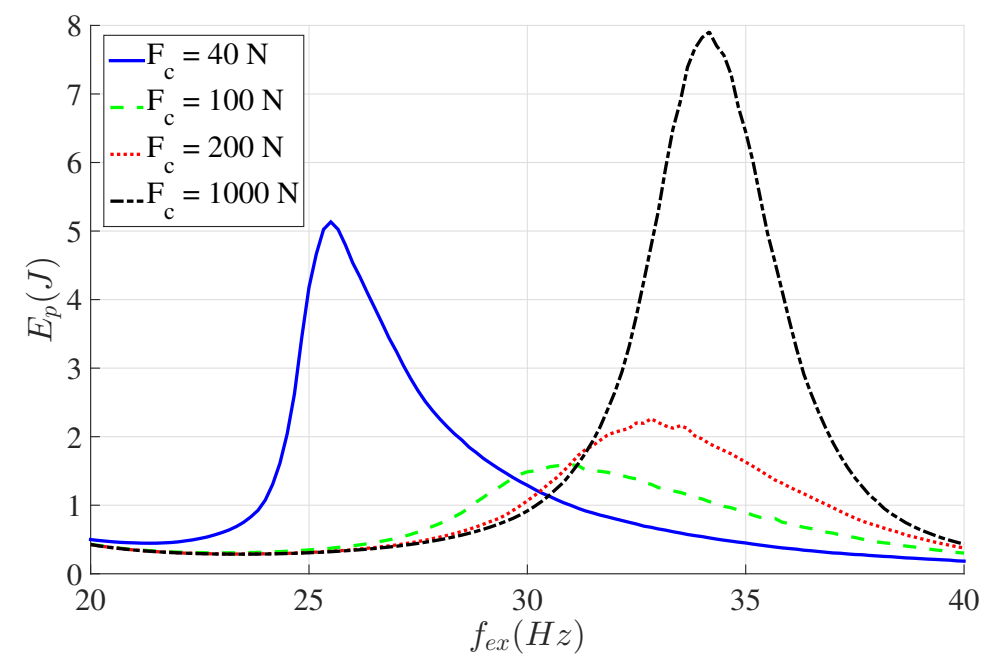

Fig. 11. Strain energy in steady state versus the excitation frequency for 4 tightening levels when the excitation force $F=100 \mathrm{~N}$

the frequencies where the strain energy reaches its maximum (Fig. 11) and where the dissipated energy reaches its maximum (Fig. 10) are not exactly the same. Indeed for the lowest values of the tightening force, the hysteresis shape changes. The horizontal line corresponding to the total slip appears at the resonance peaks. 


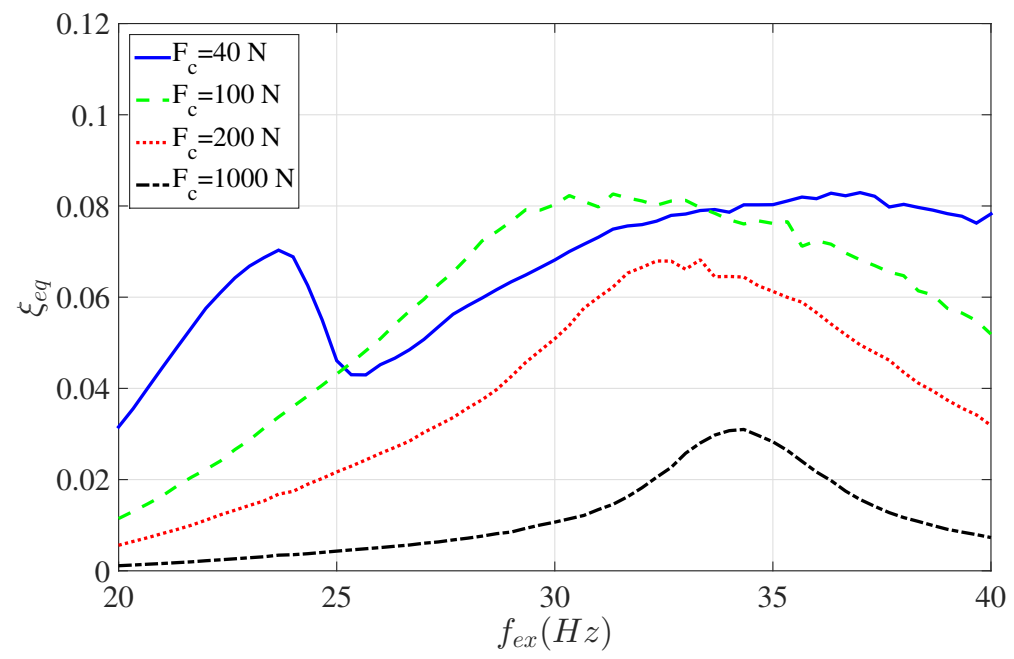

Fig. 12. Equivalent damping rates for four tightening levels when the excitation force $F=100 \mathrm{~N}$.

From this change results two peaks in the damping ratio.

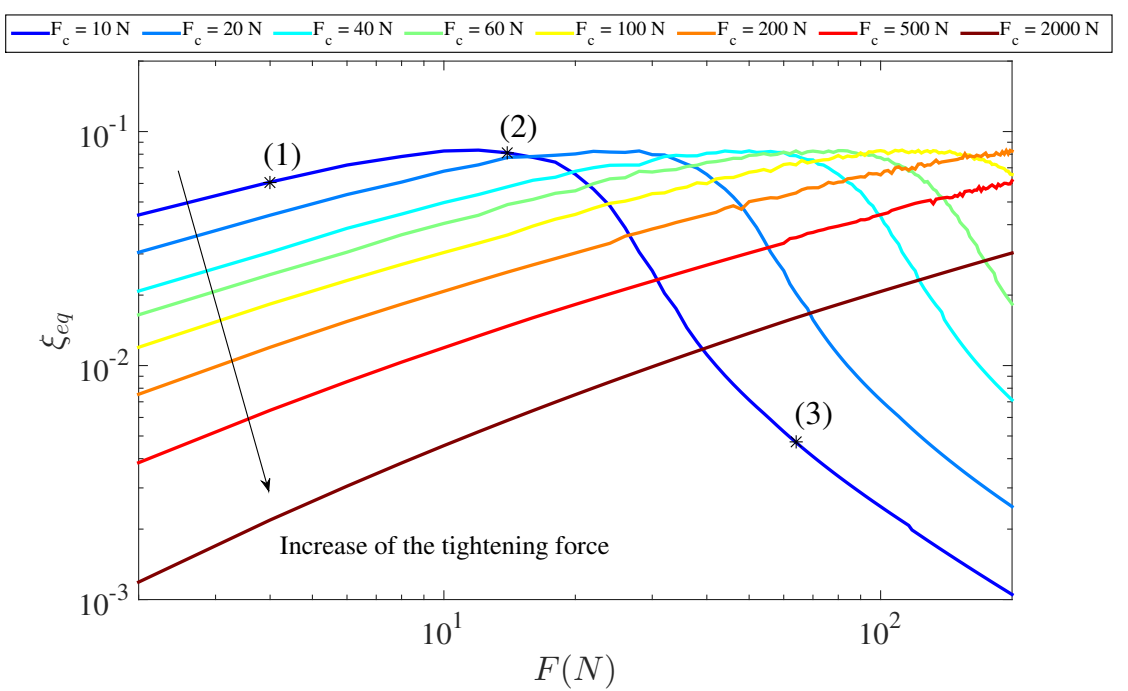

Fig. 13. Equivalent damping ratio for the second resonance frequency for $F=[2 N, 200 N]$ and for several tightening forces.

Without further study, one can conclude that the damping rate at resonance frequencies is higher when the tightening force is lower. But watching carefully to the damping evolution according to the tightening and to the excitation amplitude 
highlights an optimum tightening force $F_{c_{o p t}}$ for which the damping is higher. As the excitation force incresases, $F_{c_{o p t}}$ increases too, see Fig. 13. Therefore, it is necessary to produce a chart that gives the optimum tightening force for each excitation amplitude. The evolution of the friction induced damping at the second resonance frequency $\xi_{e q}\left(f_{r, 2}\right)$ is presented on Fig. 13. It is rather similar to experimentally observed behavior of a two overlapped bolted beams [Heller et al., 2009] and numerically computed on a lap joint [Vermot Des Roches and Balmes, 2014]. $\xi_{e q}\left(f_{0,2}\right)$ increases linearly with the logarithm of the excitation force $F$, Fig. 13, until it reaches a maximum. Then it logarithmically decreases with a greater downward slope. Moreover $\xi_{e q}\left(f_{0,2}\right)$ is inversely proportional to the transfer function $X_{1} / F$, see Fig. 15. The hysteresis loops at the resonance for the pointed conditions (1), (2) and (3), Fig. 13, are plotted on Fig. 14:

- The increase of $\left.\xi_{e q}\left(f_{0,2}\right)\right)$ corresponds to micro-slip as it is shown on Fig. 14 $(1)$

- The maximum of $\left.\xi_{e q}\left(f_{0,2}\right)\right)$ occurs just before the macro-slip transition, see Fig. 14 (2),

- The decrease of $\left.\xi_{e q}\left(f_{0,2}\right)\right)$ corresponds to macro-slip, see Fig. 14 (3).

The evolutions of the Frequency Response Function $X_{1}\left(f_{0,2}\right) / F$, for a given excitation frequency, around the second resonance frequency, according to the excitation amplitude $F$, are related to the shape of hysteresis loops, see Fig. 14 and Fig. 15:

- First $X_{i}\left(f_{0,2}\right) / F$ decreases linearly with the increase of $F$ during micro-slip,

- then $X_{i}\left(f_{0,2}\right) / F$ increases quickly during the transition from micro-slip to macro-slip,

- finally, $X_{i}\left(f_{0,2}\right) / F$ increases with a lower slope during macro slip.

From Figs. 13 and 15, ascending and descending slopes of $\xi_{e q}\left(f_{0,2}\right)$ and $X_{1}\left(f_{0,2}\right) / F$ are invariant for the different tightening force, so they are system characteristics that can be generalized for all excitations and tightening levels. It may be tricky to extract $F_{c_{\text {opt }}}$ from Figs. 13 and 15, thus Fig. 16 can be helpful for the programmation of the controller. This chart gives the first mass displacement amplitude $X_{1}$ according to the tightening force $F_{c}$ for several excitation forces $F$. For each excitation level, there is an optimum tightening force. The controller applies the tightening force according to the vibration level or to the excitation level using the chart defined on Fig. 16.

\subsection{Third control strategy}

The use of piezoelectric component allows applying a variable tightening force. The Fourier series decomposition of Dahl friction model presented by Helmick and Messner [2009] shows that the fundamental frequency is dominant in the spectrum. 

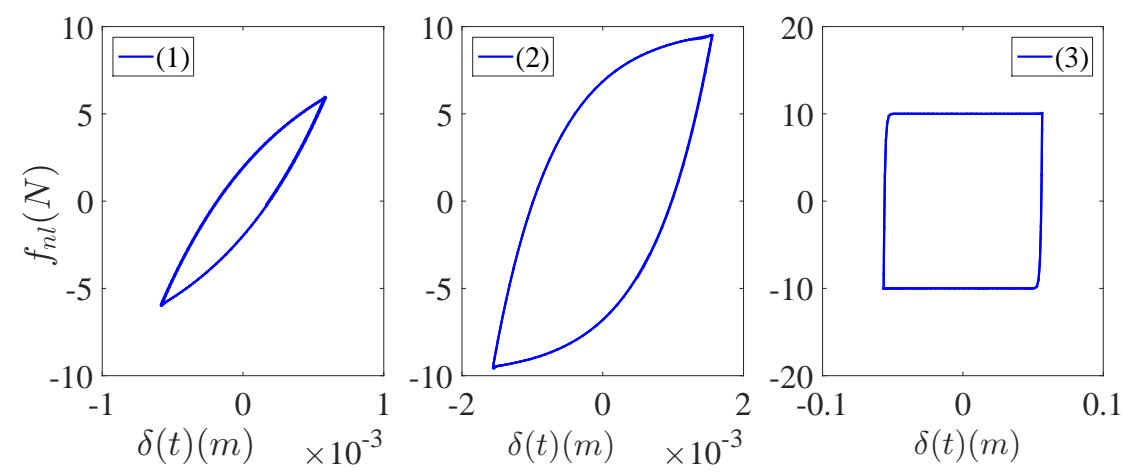

Fig. 14. Hysteresis loop at the resonance for the pointed conditions from Fig. 13.

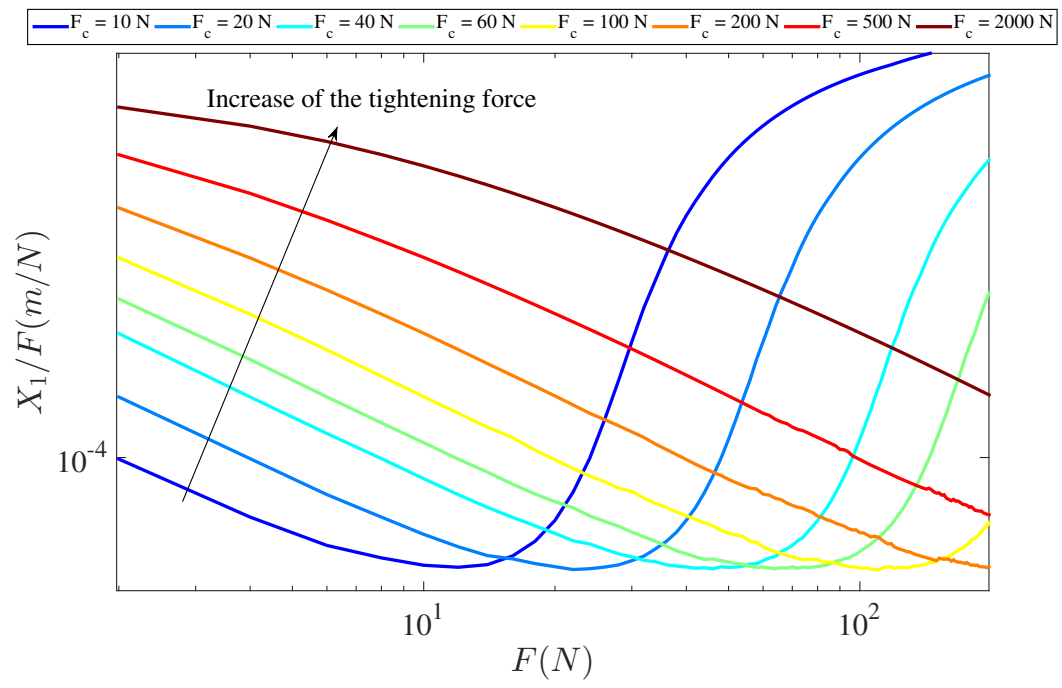

Fig. 15. FRF amplitude near the second resonance frequency according to the excitation force between $2 N$ and $200 N$ for several tightening forces.

For this reason, the proposed control law is based on a sinusoidal variation of the tightening force defined by four parameters:

- the frequency of the tightening force $\left(f_{\text {ser }}\right)$ defined as a multiple of the excitation frequency,

- the phase of the tightening force $\left(\phi_{\text {ser }}\right)$ according to the kinematics of the joint,

- the limits of the tightening force $\left(F_{c, \max }\right.$ and $\left.F_{c, \min }\right)$,

According to the previous remarks, the following definition is assumed: 


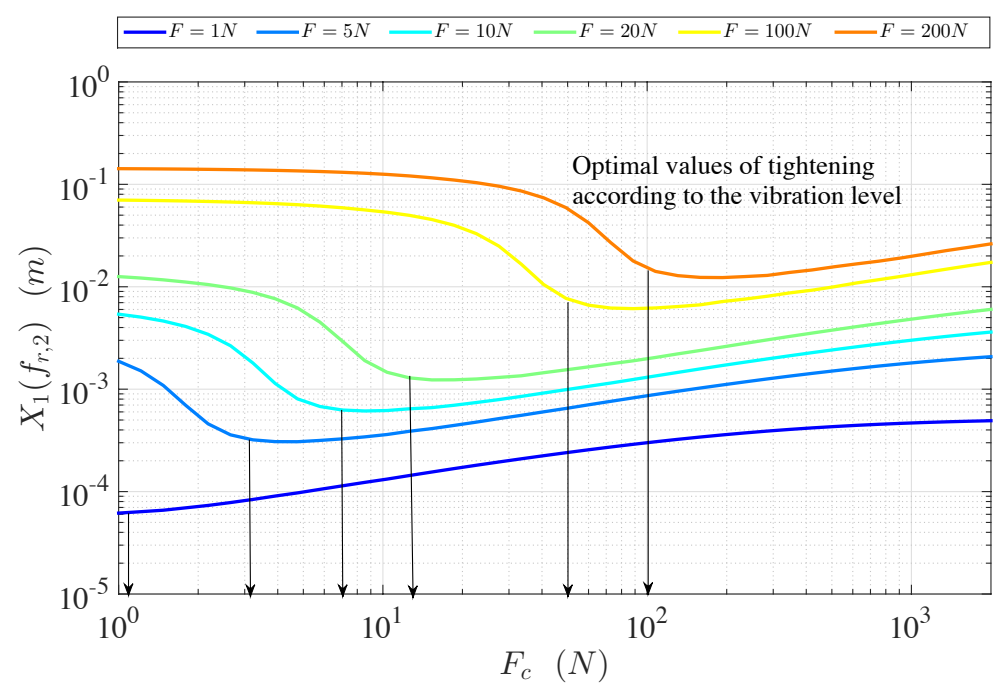

Fig. 16. First mass displacement magnitude near the second resonance frequency according to the tightening force between $2 N$ and $200 N$ for 5 levels of excitation.

$$
F_{c}(t)=\frac{F_{c, \max }+F_{c, \min }}{2}+\frac{F_{c, \max }-F_{c, \min }}{2} \cos \left(2 \pi f_{\text {ser }} t+\phi_{s e r}\right) .
$$

To initialize the control algorithm, the average tightening force is set to $F_{c_{o p t}}$ computed from the chart defined on Fig. 16, for instance $F_{c_{o p t}}(F=100 N) \cong$ $88.46 N$. To analyse the influence of the tightening frequency, the first three multiples of the excitation frequency have been tested: $f_{\text {ser }}=i f_{\text {ex }}$ with $i=1,2$ or 3 . If the excitation is sinusoidal and in steady state, the relative displacement is sinusoidal too but there is a phase shift between the relative displacement and the excitation:

$$
\delta(t)=x_{2}(t)-x_{1}(t)=\delta_{0} \cos \left(2 \pi f_{e x} t+\phi_{12}\right) .
$$

The phase of the tightening force $\phi_{\text {ser }}$ is defined according to the relative displacement phase $\phi_{12}$. Four tightening phases have been studied:

- in phase $\left(\phi_{\text {ser }}=\phi_{12}\right)$;

- advanced quadrature-phase $\left(\phi_{\text {ser }}=\phi_{12}+\pi / 2\right)$;

- retarded quadrature-phase $\left(\phi_{\text {ser }}=\phi_{12}-\pi / 2\right)$;

- in opposed phase $\left(\phi_{\text {ser }}=\phi_{12}+\pi\right)$.

The displacement magnitudes of the first mass are plotted on Figs. 17 and 18 according to the excitation frequency. $f_{\text {ser }}$ and $\phi_{\text {ser }}$ affect the vibration magnitudes and the resonance frequency especially in the cases $\phi_{\text {ser }}=\phi_{12} \pm \pi / 2$. The sensitivity is higher for $f_{\text {ser }}=2 f_{\text {ex }}$. Thus, the suitable parameters of the law control are 

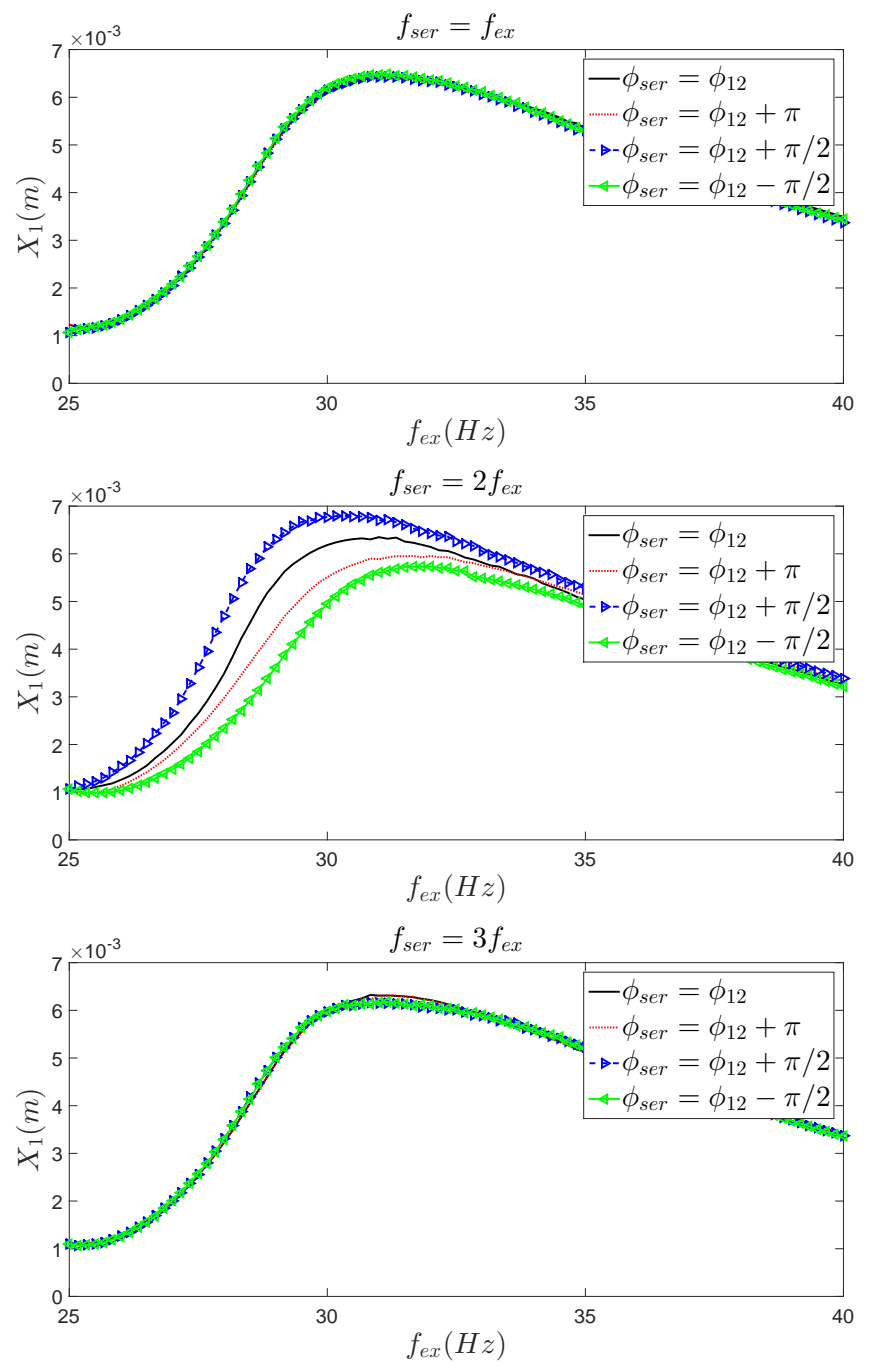

Fig. 17. First mass displacement magnitude according to the excitation frequency when, $F_{c, \min }=$ $68.5 \mathrm{~N}, F_{c, \max }=108.5 \mathrm{~N}, F=100 \mathrm{~N}$ and $(\mathrm{Top}) f_{\text {ser }}=f_{\text {ex }}$ (Middle) $f_{\text {ser }}=2 f_{\text {ex }}$, (Down) $f_{\text {ser }}=3 f_{\text {ex }}$.

$f_{\text {ser }}=2 f_{\text {ex }}$ and $\phi_{\text {ser }}=\phi_{12}-\pi / 2$. The second step of the control algorithm is to set the tightening frequency according to the vibration frequency and to set the phase according to the vibration phase.

The third step of the control algorithm is to set the limits of tightening $F_{c, \max }$ and $F_{c, \text { min }}$. Increasing the gap between the limit values of the tightening force results a vibration reduction for all the excitation domain (damping effect) and a shift on the right of the resonance frequency (stiffening effect) (Fig. 19). For the largest gap levels, a bifurcation occurs (see Figs. 19 and 23). This means that in a 

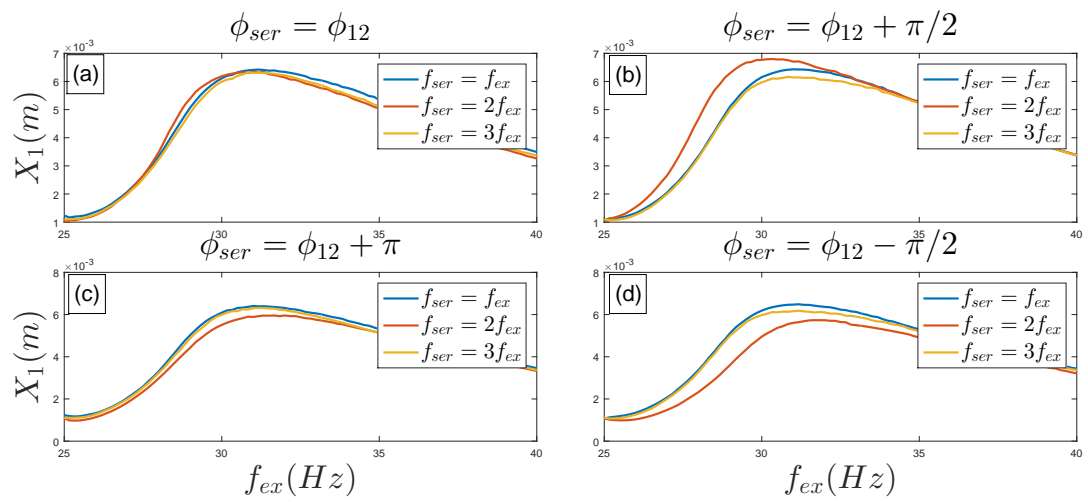

Fig. 18. First mass displacement amplitude according to the excitation frequency when, $F_{c, \text { min }}=$ $68.5 N, F_{c, \max }=108.5 \mathrm{~N}, F=100 \mathrm{~N}$ and (a) $\phi_{s e r}=\phi_{12}$, (b) $\phi_{\text {ser }}=\phi_{12}+\pi / 2$, (c) $\phi_{\text {ser }}=\phi_{12}+\pi$, (d) $\phi_{\text {ser }}=\phi_{12}-\pi / 2$.

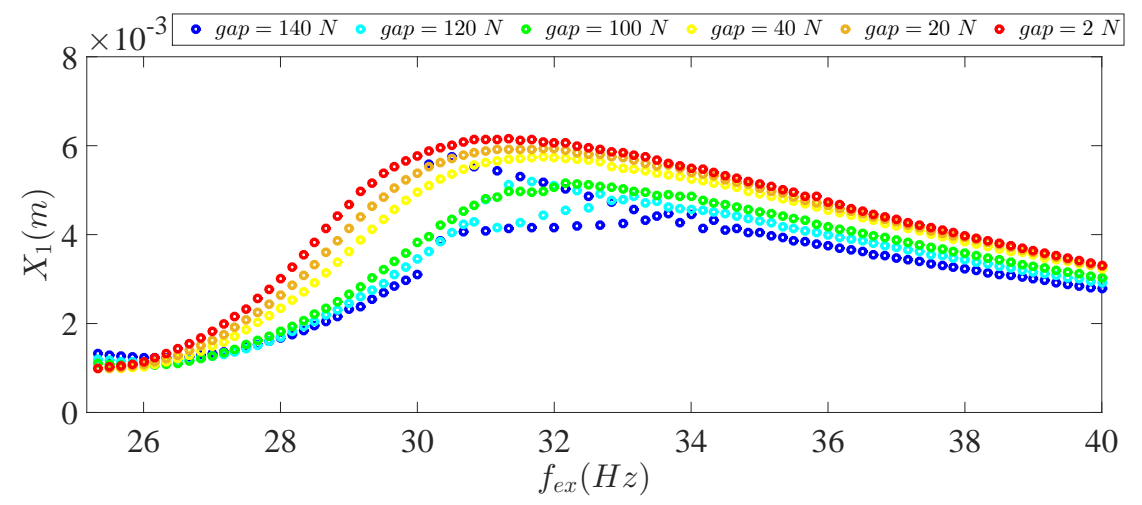

Fig. 19. Amplitude of the first mass in the neighborhood of the second resonance frequency for an excitation force $F=100 \mathrm{~N}$ and for a six level of gap between $F_{c, \min }$ and $F_{c, \max }$ in the case of retarded-phase and quadrature components $\phi_{s e r}=\phi_{12}-\pi / 2$ and $f_{s e r}=2 f_{e x}$.

frequency bandwidth, two solutions are available. It may be caused by the computation of the phase $\phi_{12}$ as for a given excitation frequency, the phase is calculated from the previous excitation frequency. The bifurcation can also be induced by the nonlinearity itself and the occurrence of a bifurcation according to the excitation frequency. Indeed, the large gaps between the tightening limits increase the nonlinearity effect. In this case the vibration amplitude oscillation can take one of two values chaotically (see Fig. 23 ). The oscillations of the vibration amplitude are not caused due an unstable source of the active control of the normal force. From the same figure, one can observe that the hysteresis loop is strongly modulated for the upper branch. Moreover, the area of the hysteresis loop, i.e. the energy dissipated is lower, that can explain the fact that this branch corresponds to higher amplitudes. 
The system remains inherently passive or semi-active for each frequency and amplitude of excitation. Anyway, even if a bifurcation occurs, the amplitudes are still lower than for the constant tightening case (see Figs. 19 and 24).

The proposed control law has shown its effectiveness for all the excitation levels, (see Fig. 23). Figs. 20 and 21, show, the evolution of the tightening force, the relative displacement and the resulting force of the Jenkins'cell, for two different gap values. In order to compare the shapes of the curves, they are normalized. On the right part of each figure, the hysteresis loop $f_{n l}$ versus $\delta$ is plotted. Compared to the case of a constant tightening force (the usual shape of Dahl's loop), the hysteresis loop is significantly modulated mainly when the gap between the limit values of the tightening force is great, see Fig. 22. For the highest gap (see Fig. 22), the coupling stiffness increases, just after the sign of the relative velocity changes, until the tightening force tends to zero; then the stiffness is lower up to the next sign change.

Finally, the third control strategy is based on a sinusoidal tightening force characterized by:

- a tightening frequency equal to twice the excitation frequency $\left(f_{\text {ser }}=2 f_{\text {ex }}\right)$;

- a $-\pi / 2$ phase between the tightening and the relative displacement $\left(\phi_{\text {ser }}=\right.$ $\left.\phi_{12}-\pi / 2\right)$;

- an average tightening amplitude force equal to the optimum tightening force, see second control strategy $\left(\left(F_{c, \max }+F_{c, \min }\right) / 2=F_{c_{o p t}}\right)$;

- a gap between the limits of tightening as large as possible without causing a bifurcation $\left(\right.$ gap $\left._{\max }\right)$;

The tightening law is expressed as follows:

$$
F_{c}(t)=F_{c_{o p t}}+\frac{g a p_{\max }}{2} \cos \left(4 \pi f_{e x} t+\phi_{12}-\pi / 2\right)
$$

With this formulation the results are improved in comparison with those obtained in the case of an optimum constant tightening force as it can be seen in Fig. 22 and Fig. 24.

From Fig. 24, the vibration amplitudes $\left(X_{1} / F\right)$ are presented for the two cases of tightening force; constant and sinusoidal. For the excitation $F=100 \mathrm{~N}$, the sinusoidal control law is more effective than the optimal tightening force for the whole frequency band in the vicinity of the second resonant frequency. Another advantage of this control law is that it is effective for all vibration levels $(F=1 N$ to $400 N$ in Fig. 24). For all the excitation levels, the control of the tightening force has the tendency to force the resonant frequency and the $X_{1} / F$ ratio to the same values (see Fig. 24). Hence, knowing the amplitude vibration of a single level of excitation, the vibration response can be estimated for any level of excitation when the suitable parameters of the tightening force are applied. 

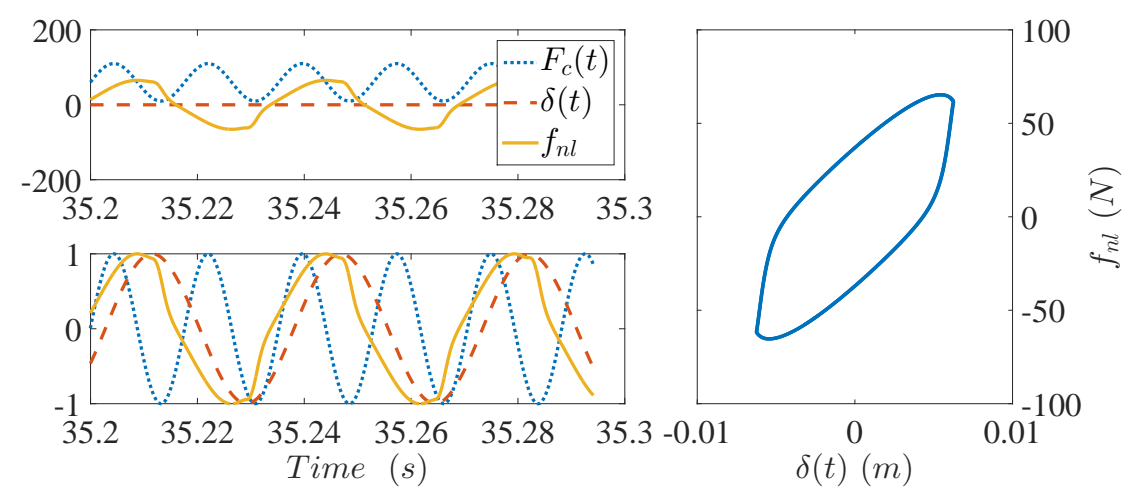

Fig. 20. (left) Comparison of the shapes of the tightening force $\left(F_{c}(t)\right)$, the displacement relative $(\delta(t))$ and the resulting force of Jenkins'cell $\left(f_{n l}(t)\right)$. (right) Presentation of the hysteresis loop $f_{n l}(\delta)$ in case of $F_{c, \min }=10 N, F_{c, \max }=110 N, F=100 N, \phi_{s e r}=\phi_{12}-\pi / 2, f_{\text {ser }}=2 f_{\text {ex }}$ and $f_{e x}=28,33 H z$.
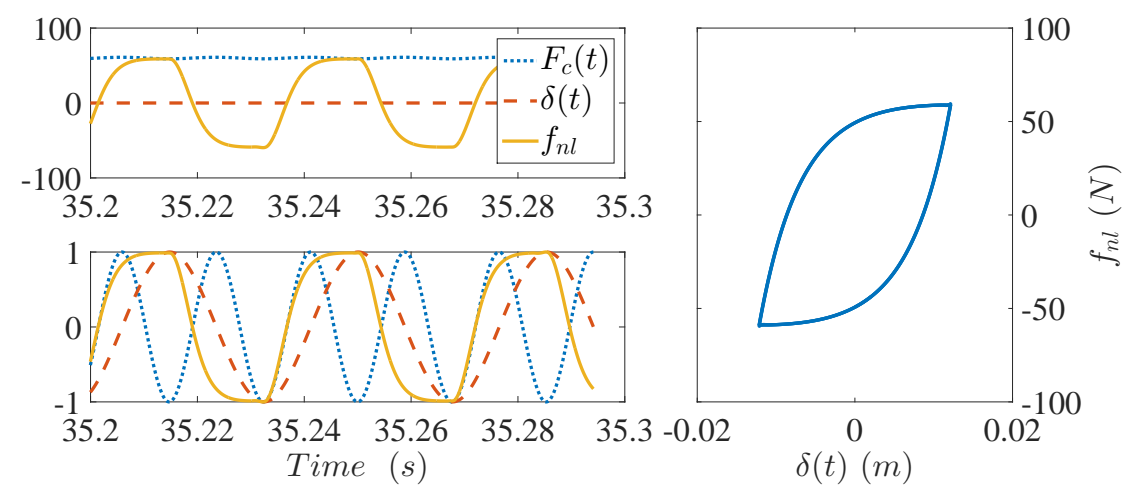

Fig. 21. (a) Comparison of the shapes of the tightening force $\left(F_{c}(t)\right)$, the displacement relative $(\delta(t))$ and the resulting force of Jenkins'cell $\left(f_{n l}(t)\right)$, (b) and presentation of the hysteresis loop $f_{n l}(\delta)$ in case of $F_{c, \min }=59 \mathrm{~N}, F_{c, \max }=61 \mathrm{~N}, \mathrm{~F}=100 \mathrm{~N}, \phi_{\text {ser }}=\phi_{12}-\pi / 2, f_{\text {ser }}=2 f_{\text {ex }}$ and $f_{e x}=28,33 \mathrm{~Hz}$.

\section{4. conclusions}

Three control strategies have been evaluated for the mitigation of steady state vibrations under harmonic excitation. The two first ones are interesting because they are based on a quasi constant tightening force calculated from the excitation frequency and the excitation amplitude. However their efficiency is less interesting than the the third control strategy which is based on a harmonic evolution of the tightening force calculated from the frequency and the amplitude of the vibrations and shifted of $\pi / 2$ negative phase. If a piezoelectric actuator was used to change the tightening force, as it behaves as a capacitance, the two first control strategies 


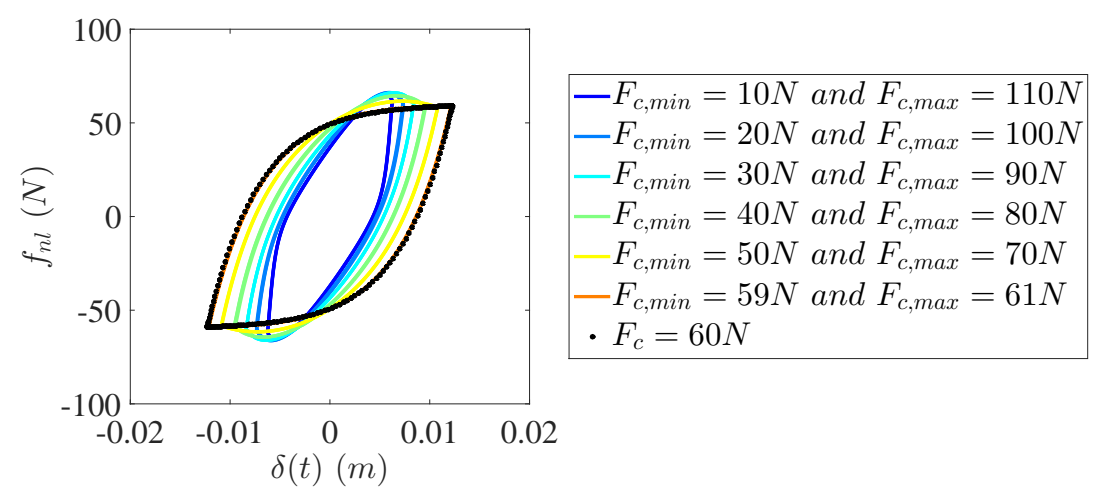

Fig. 22. Comparison of the hysteresis loop when the tightening force is sinusoidal (solid line) and when it is constant (dot line) in case of $F=100 \mathrm{~N}, \phi_{\text {ser }}=\phi_{12}-\pi / 2, f_{\text {ser }}=2 f_{\text {ex }}$ and $f_{e x}=28,33 \mathrm{~Hz}$.

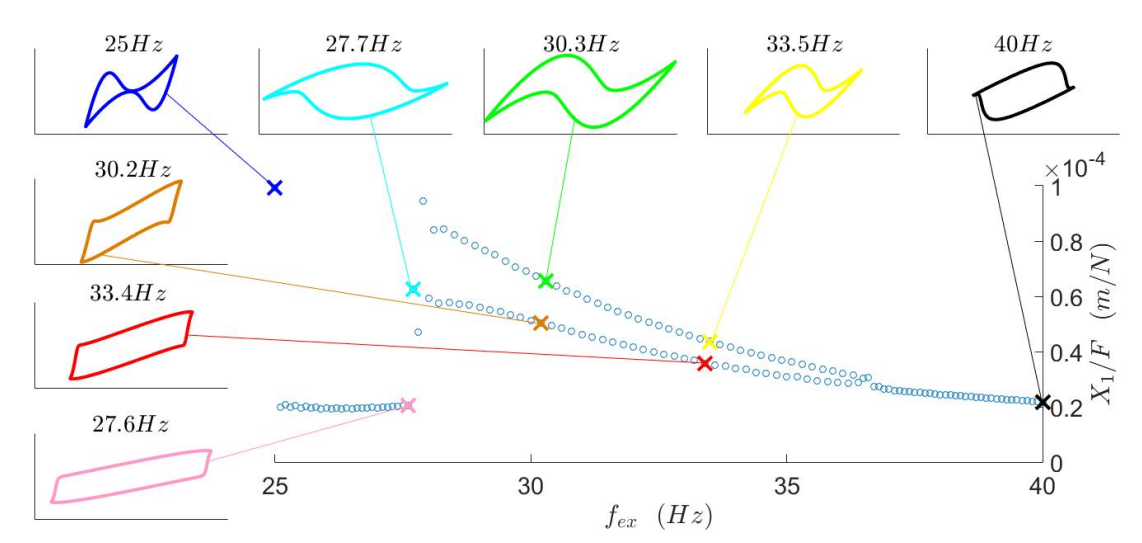

Fig. 23. Evolution of $X_{1} / F$ and the hysteresis loop when $F_{c}$ follows our control law, $F_{1}=100 N$, $F_{c_{\max }}=159 \mathrm{~N}$ and $F_{c_{\min }}=1 \mathrm{~N}$. Link between both bifurcation branches and the hysteresis loop

would consume less electric power. This last point should be assessed in a future study.

\section{Control strategies for transient vibrations}

To test the efficiency of the control strategies on transient vibrations, the modal damping is canceled $(\xi=0)$. Thus the 2-DOFs system is given by:

$$
\left\{\begin{array}{l}
{\left[\begin{array}{cc}
m_{1} & 0 \\
0 & m_{2}
\end{array}\right]\left(\begin{array}{l}
\ddot{x} \\
\ddot{y}
\end{array}\right)+\left[\begin{array}{cc}
k_{1}+k_{12} & -k_{12} \\
-k_{12} & k_{1}+k_{12}
\end{array}\right]\left(\begin{array}{l}
x \\
y
\end{array}\right)=\left(\begin{array}{c}
-f_{n l} \\
f_{n l}
\end{array}\right)} \\
x_{1}(t=0)=x_{2}(t=0)=0 \\
\dot{x}_{1}(t=0) \neq 0, \dot{x}_{2}(t=0) \neq 0
\end{array} .\right.
$$

The initial conditions are chosen in order to excite the second mode which is 

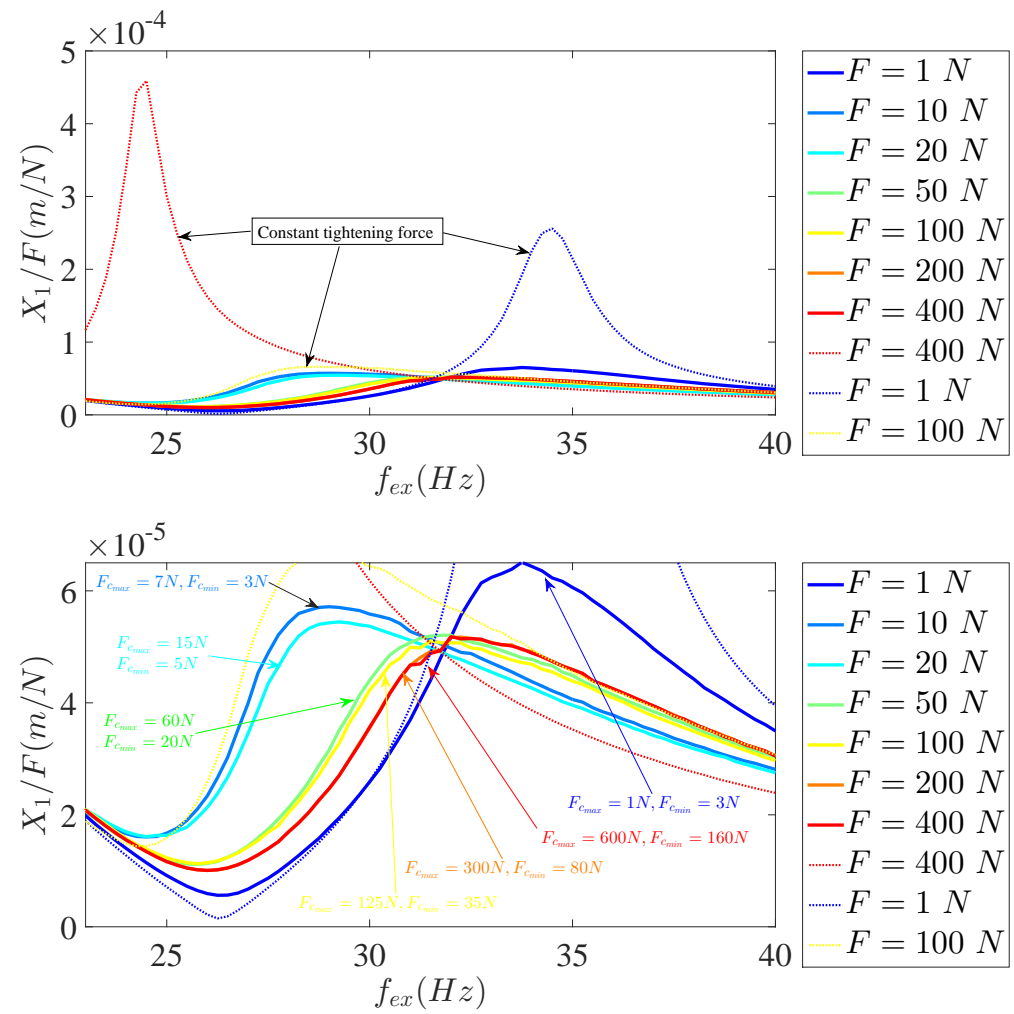

Fig. 24. Comparison of $X_{1} / F$ for the two cases: dotted line $F_{c}$ is constant $\left(F_{c}=60 N\right)$ and continuous line $F_{c}$ follows our control law for several levels of excitation.

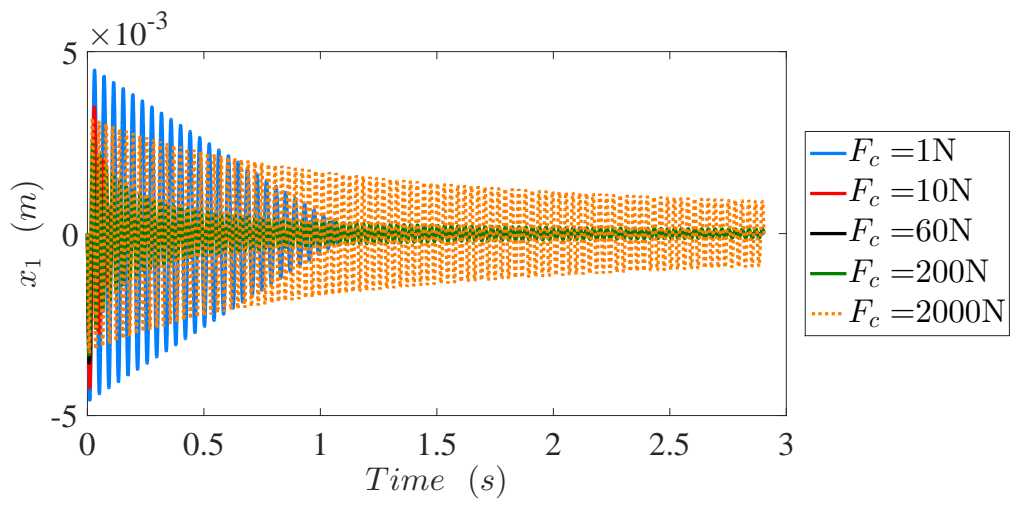

Fig. 25. Comparison of the constant tightening force effect on the attenuation of the vibration amplitudes. 
the more coupled, see section 2. Fig. 25 presents the amplitude decay for different constant tightening forces. During the first time steps, the lowest tightening forces are responsible for the highest amplitudes due to the lack of rigidity. While during the last time steps, the smaller the tightening force, the smaller the amplitude. The optimal tightening force is a good compromise but the magnitude tends to a finite value when the time tends to the infinity. From this first observation, applying a constant tightening force is not optimal for the transient vibrations. The simplified law inspired from [Dupont et al., 1997] and [Gaul and Nitsche, 2000] works on the impulse excitation, is as follow:

$$
F_{c}(t)=\left\{\begin{array}{l}
F_{c, \max } \text { if } \dot{\delta}(t)>0 \\
F_{c, \min } \text { elsewhere }
\end{array} .\right.
$$

This control strategy minimizes the feedback of frictional energy to the system. The system is mainly sensitive to $F_{c, \text { min }}$. This means that the damping is higher when $F_{c, \min }$ is low (see Fig. 26). In the initial phase, it is necessary to increase the gap between the tightening levels to have the largest damping. $F_{c, \max }$ has no effect on the long-term behavior. Finally, applying an alternated tightening force, is more efficient than applying a constant tightening force particularly for the first phase (large vibration amplitudes) and the stabilization phase (low vibration amplitudes) (Fig. 27). In [Dupont et al., 1997] and [Gaul and Nitshe, 2000], the authors introduced a "boundary layer of thickness $\epsilon$ " to regularize the control discontinuity introduced by the relative-velocity sign change. Increasing $\epsilon$ results a decreasing of the induced frictional damping. However, as it is shown by the computation algorithm in Fig. 29, increasing $\epsilon$ is desirable because in our case we aim the fast zeroing of the relative displacement while avoiding the variation of the tightening force. Inserting the constant boundary layer of thickness $\epsilon$ improves the law (14) as follow:

$$
F_{c}(t)=\left\{\begin{array}{cl}
F_{c, \text { max }} & \text { for } \quad \dot{\delta}(t) \geq \epsilon \\
\frac{F_{c, \text { max }}+F_{c, \text { min }}}{2}+\frac{F_{c, \text { max }}-F_{c, \text { min }}}{2} \cos \left(\frac{\pi \dot{\delta}}{\epsilon}\right) & \text { for } 0<\dot{\delta}(t)<\epsilon . \\
F_{c, \text { min }} & \text { for } \quad \dot{\delta}(t) \leq 0
\end{array}\right.
$$

The intermediate layer level $\epsilon$ has no effect in the first phase of excitation and even in the stabilized phase, the impact on amplitude vibration is very low. But it is important to increase the intermediate layer level $\epsilon$ without exceeding the maximum relative velocity value to reduce the modulation of the tightening force as shown on Fig. 28. In a real system we cannot know the maximum relative speed, so we can calculate the intermediate layer of each level $\epsilon$ only after the sensors signals acquisition. Therefore, $\epsilon(t)$ is defined as a function of the relative displacement $\epsilon(t)=G(\dot{\delta}(t))$. The computation algorithm, see Fig. 29, is used to get the results 


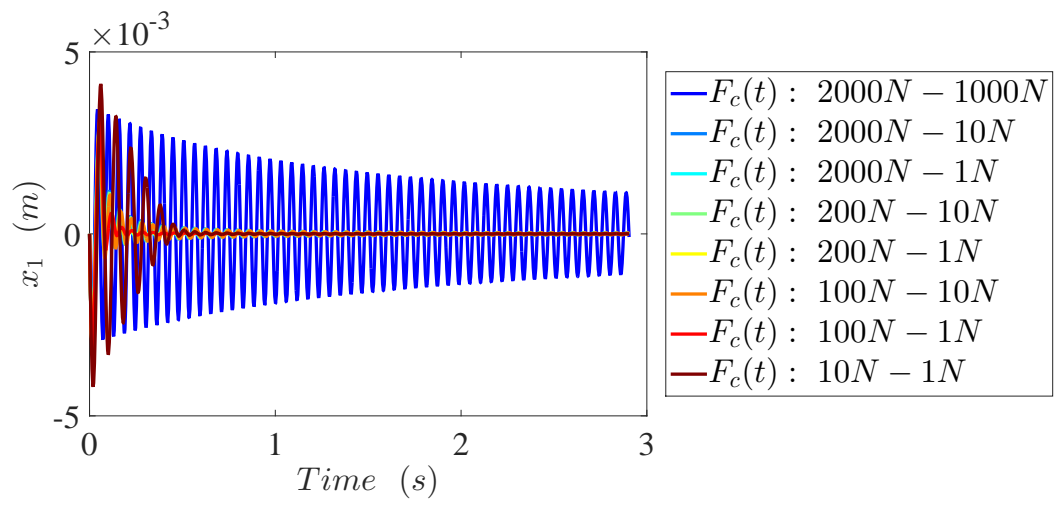

Fig. 26. Comparison of the alternated tightening force effect on the attenuation of the vibration amplitudes.

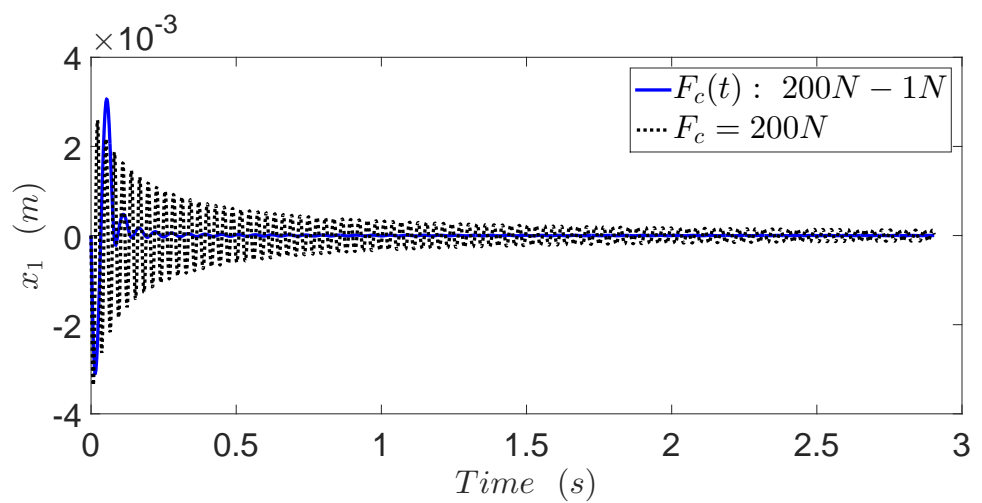

Fig. 27. Magnitude of the first mass displacement for: (solid line) alternated tightening force and (dotted line) constant tightening force.

presented in Fig. 30.

$$
F_{c}(t)=\left\{\begin{array}{cl}
F_{c, \text { max }} & \text { for } \quad \dot{\delta}(t) \geq G(\dot{\delta}(t)) \\
\frac{F_{c, \text { max }}+F_{c, \text { min }}}{2}+\frac{F_{c, \text { max }}-F_{c, \text { min }}}{2} \cos \left(\frac{\pi \dot{\delta}}{G(\dot{\delta}(t))}\right) & \text { for } 0<\dot{\delta}(t)<G(\dot{\delta}(t)) . \\
F_{c, \text { min }} & \text { for } \quad \dot{\delta}(t) \leq 0
\end{array}\right.
$$

The implementation of a variable intermediate layer level $\epsilon(t)$, minimizes the energy need for the control of the tightening force and eliminates unnecessary variation of the tightening force (Fig. 30b) without losing the effectiveness of the control law (14) (Fig. 30a). 

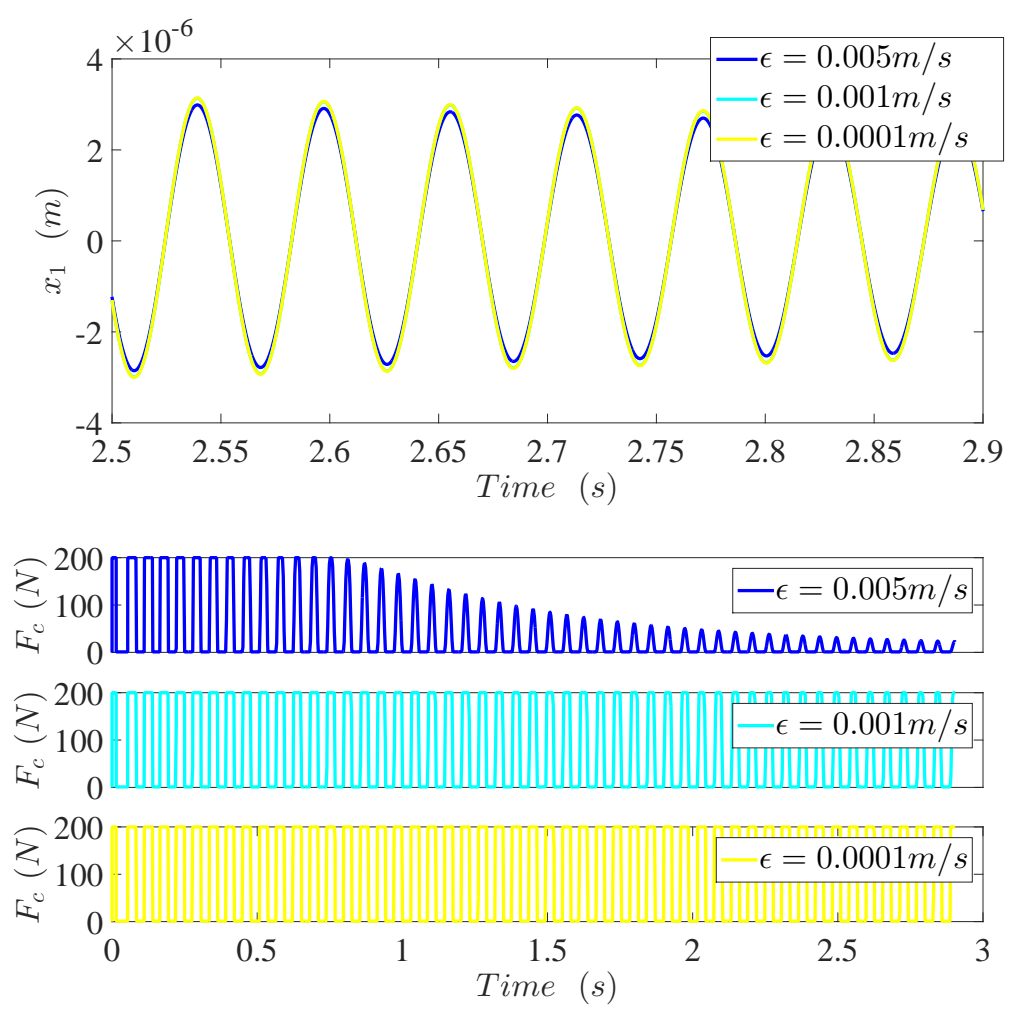

Fig. 28. (a) The first mass vibration amplitude at the stabilization phase and (b) the evolution of the tightening force for three intermediate layer level ?in the case of $F_{c, \max }=200 \mathrm{~N}$ and $F_{c, \min }=1 \mathrm{~N}$.

\section{Conclusion}

In this paper we define the "joint coupling coefficient" as a performance indicator of the joint ability to reduce vibrations. This coefficient highlights the sensitivity of the structure to the joint tightening force and to the joint loading amplitudes. The joint coupling coefficient has been evaluated in this work in order to choose the most coupled mode and to define the best set of parameters.

Three tightening laws are proposed to control steady state vibrations under harmonic load. The first one consists in switching between two values of the tightening force. This kind of control is very close to the switching control proposed by Badel et al. but the physics involved is different. This control is more efficient when the joint coupling coefficient is great. The second law consist in applying an optimum constant tightening force for each level of excitation. The third law is denoted as "a phase control law", and is more effective than the second law with an optimum constant tightening force. This law depends on the amplitudes and frequencies of the excitation and the relative displacement. 


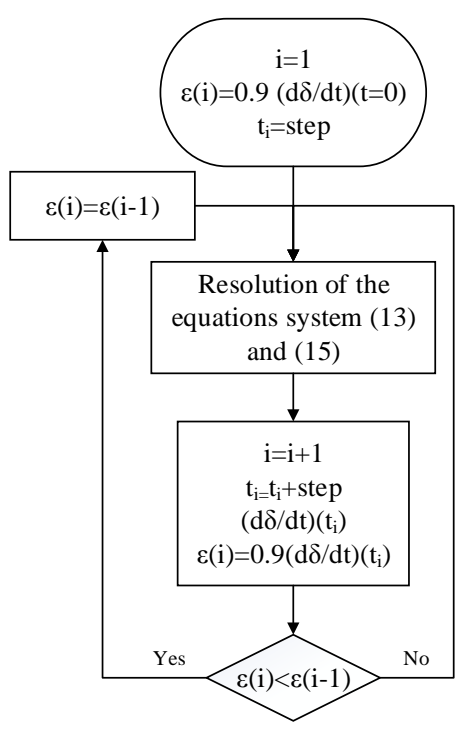

Fig. 29. Compute algorithm.

For transient vibrations, we ameliorate the control strategy referenced in the literature. The improvement avoids the periodic change in the tightening force and thus the cyclic fatigue and the electrical energy consumption.

\section{Acknowledgements}

The authors are grateful to the Fonds Unique Interministériel (FUI) for their financial support of this project (CLIMA - FUI 19).

\section{References}

Badel, A., Sebald, G., Guyomar, D., Lallart, M., Lefeuvre, E., Richard, C., Qiu, J. [2006] "Piezoelectric vibration control by synchronized switching on adaptive voltage sources: Towards wideband semi-active damping," The Journal of the Acoustical Society of America, 119(5), 2815-2825.

Bliman, P. [1992] "Mathematical study of the dahl's friction mode," European Journal of Mechanics A:Solids 11(6), 835-348.

Chevallier, G., Ghorbel, S. and Benjeddou, A. [2008] "A benchmark for free vibration and effective coupling of thick piezoelectric smart structures," Smart Materials and Structures 17(6), 065007.

Dahl, P. R. [1968] "A solid friction model," Tech. rep., DTIC Document.

Deaner, B. J., Allen, M. S., Starr, M. J., Segalman, D. J., and Sumali, H. [2015] "Application of Viscous and Iwan Modal Damping Models to Experimental Mea- 

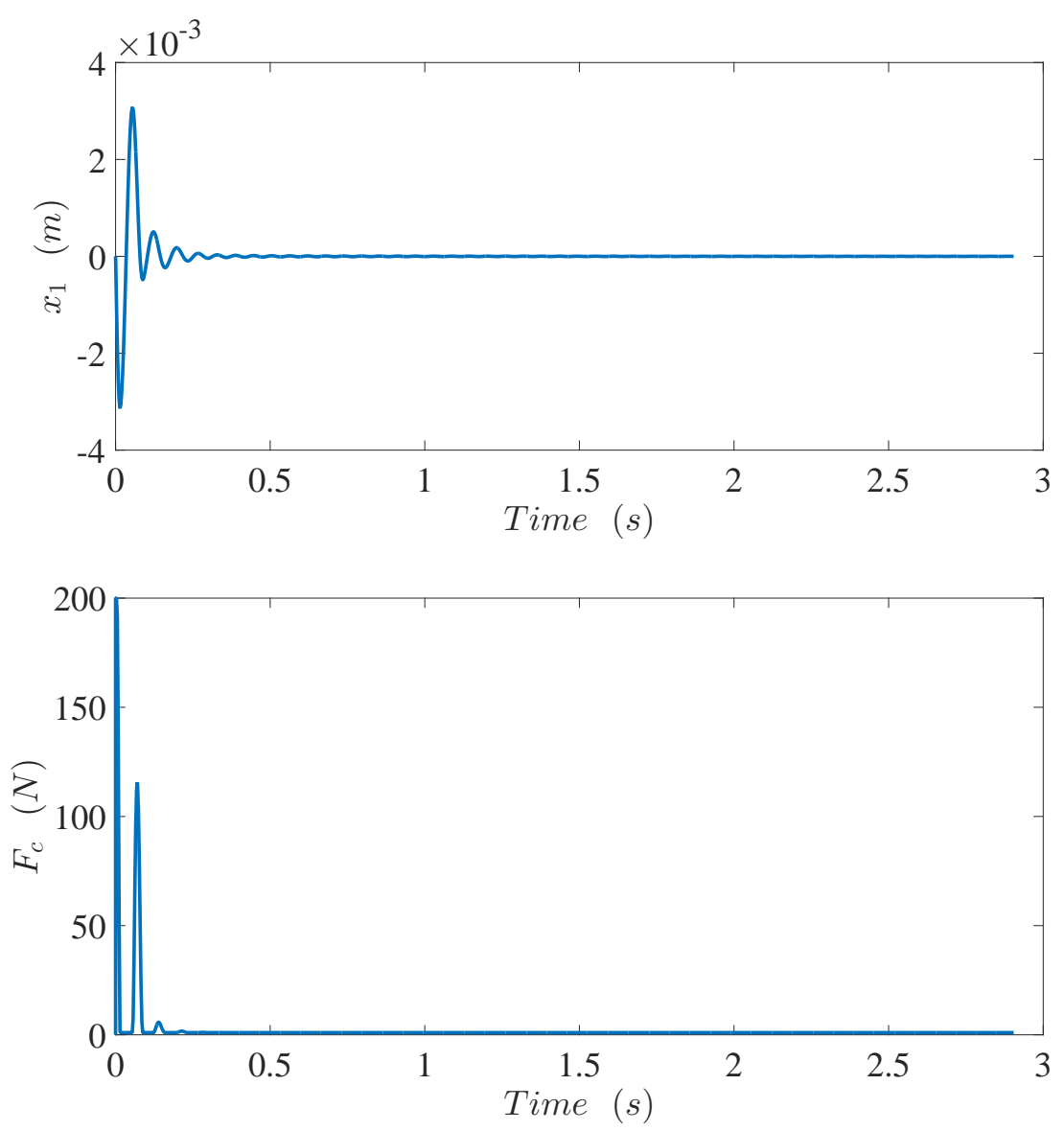

Fig. 30. (a) The first mass vibration amplitude and (b) the evolution of the tightening force for the variable intermediate layer level $\epsilon(t)$ in the case of $F_{c, \max }=200 \mathrm{~N}$ and $F_{c, \min }=1 \mathrm{~N}$.

surements From Bolted Structures," Journal of Vibration and Acoustics 137(2), 021012.

De Wit, C. C., Olsson, H., Astrom, K. J., and Lischinsky, P. [1995] "A new model for control of systems with friction," Automatic Control, IEEE Transactions on 40(3), 419-425.

Dion, J.-L., Chevallier, G., and Peyret, N. [2013] "Improvement of measurement techniques for damping induced by micro-sliding," Mechanical Systems and Signal Processing 34(1), 106-115.

Dupont, P., and Stokes, A. [1995] "Semi-active control of friction dampers, Decision and Control, 1995., Proceedings of the 34th IEEE Conference on, Vol. 4, pp. 3331-3336.

Dupont, P., Kasturi, P., and Stokes, A. [1997] "Semiactive control of friction dampers," Journal of sound and vibration 202(2), 203-218. 
Festjens, H., Chevallier, G., and Dion, J. L. [2014] "Nonlinear model order reduction of jointed structures for dynamic analysis," Journal of Sound and Vibration 333(7), 2100-2113.

Gaul, L., and Lenz, J. [1997] "Nonlinear dynamics of'structures assembled by bolted joints," Acta Mechanica 125(1-4), 169-181.

Gaul, L., Lenz, J., and Sachau, D. [1998] "Active damping of space structures by contact pressure control in joints," Journal of Structural Mechanics 26(1), 81100.

Gaul, L., and Nitsche, R. [2000] "Friction control for vibration suppression," $\mathrm{Me}$ chanical Systems and Signal Processing 14(2), 139-150.

Gaul, L., Albrecht, H. and Wirnitzer, J. [2004] "Semi-active friction damping of large space truss structures," Shock and Vibration 11(3-4), 173-186.

Gaul, L., Hurlebaus, S., Albrecht, H., and Wirnitzer, J. [2005] "Controlled friction damping using optimally located structural joints," In Control and Observer Design for Nonlinear Finite and Infinite Dimensional Systems, Springer, Berlin, Heidelberg ,pp. 335-350.

Hammami, C., Balmes, E., and Guskov, M. [2016] "Numerical design and test on an assembled structure of a bolted joint with viscoelastic damping," Mechanical Systems and Signal Processing 70, 714-724.

Heller, L., Foltête, E., and Piranda, J. [2009] "Experimental identification of nonlinear dynamic properties of built-up structures," Journal of Sound and Vibration 327(1), 183-196.

Helmick, D., and Messner, W. [2009] "Describing function analysis of dahl model friction," In American control conference, pp. 814-819.

Karim, Y., and Blanzé, C. [2014] "Vibration reduction of a structure by design and control of a bolted joint," Computers and Structures 138, 73-85.

Lane, J., Ferri, A., and Heck, B. [1992] "Vibration control using semi-active frictional damping," Friction Induced Vibration, Chatter, Squeal and Chaos DE 49, $165-171$.

Mascarenas, D. D., Park, G., and Farrar, C. R. [2005] "Monitoring of bolt preload using piezoelectric active devices," In Nondestructive Evaulation for Health Monitoring and Diagnostics, International Society for Optics and Photonics, pp. $129-136$.

Mascarenas, D., Todd, M., Park, G., and Farrar, C. [2006] "Remote inspection of bolted joints using rfid-tagged piezoelectric sensors," In IMAC-XXIV: Conference and Exposition on Structural Dynamics, St. Louis, MO, USA.

Pennestri, E., Valentini, P., and Vita, L. [2007] "Multibody dynamics simulation of planar linkages with dahl friction," Multibody System Dynamics 17(4), 321-347.

Peyret, N, Dion, J.-L., Chevallier, G., Argoul, G. [2010] "Micro-slip induced damping in planar contact under constant and uniform normal stress," International Journal of Applied Mechanics 02(2), 281-304.

Shen, J.-C., and Yan, C.-C. [2013] "Tracking control of a precision stage with in- 
tegral sliding-mode friction estimator," Microsystem technologies 19(11), 17311736 .

Stammers, C., and Sireteanu, T. [1998] "Vibration control of machines by use of semi-active dry friction damping," Journal of Sound and Vibration 209(4), 671684.

Vermot Des Roches, G., and Balmes, E. [2014] "Understanding friction induced damping in bolted assemblies through explicit transient simulation," International Conference on Noise and Vibration Engineering, pp. ID-360, 